Propellant Sloshing Parameter Extraction from CFD Analysis

\author{
Dr. H. Q. Yang \\ CFD Research Corp.-Jacob \\ ER42 \\ NASA MSFC \\ and \\ Mr. John Peugeot \\ ER42 \\ NASA MSFC
}

An Extended Abstract Submitted to AIAA Joint Propulsion Conference July 25-28, 2010

Nashville, TN 


\section{INTRODUCTION}

Propellant sloshing is a potential source of disturbance that is critical to the stability of launch vehicles. The driving sloshing forces of the propellant masses come from the lateral disturbance, oscillatory thrust force, or angular rotation of the vehicle during the powered phase of the flight. Sloshing is expected to occur during vehicle taxi, takeoff, engine shut off, and flight maneuvers. Fluid motion produced during these operations can circulate sub-cooled liquid fuel near the liquid vapor interface resulting in increased condensation and corresponding pressure collapse. Conversely, liquid contact on hot tank walls caused by sloshing can result in rapid vaporization and subsequent rapid pressure rise. Understanding the dynamic behavior and the response of cryogenic propellants of Liquid Hydrogen (LH2) and Liquid Oxygen (LOX) tanks under spacecraft flight operations (such as engine restart, oscillatory thrust and oscillatory side forces) is extremely important during the vehicle design. In addition, the proper orientation of propellant is necessary for improved performance of the tank vent system, to ensure sufficient liquid propellant for engine firing and proper thermal conditions in feed lines prior to ignition, and to minimize vehicle disturbances and avoid excessive demands on the attitude controls.

Sloshing is a wave phenomenon, and is driven by gravitational forces, surface tension, and surface kinematics. To take all the detailed dynamics into account in a vehicle dynamic modeling is very time consuming. Previously, a spring mass damper model has been used for Guidance, Navigation and Control Analysis (GN\&C). Using first principle based Newtonian fluid dynamics; NASA Marshall Space Flight Center has developed a high fidelity Computational Fluid Dynamics (CFD) model for simulation and analysis of sloshing phenomenon. This model takes into account all the essential physics of fluid-gas interface, effect of gravitational force, surface tension and surface kinematics, to model the tank slosh using the acceleration forces provided by GN\&C.

The objective of this study is to validate CFD extracted mechanical parameters (such as sloshing mass, sloshing frequency, and sloshing mass center) against theoretical solution derived in 1960's for the simple cylindrical tanks and against experimental data taken during the 1960's for more complex geometries

\section{ANALYSIS MODELING APPROACH}

\section{1. $\quad$ Fluid Dynamics Analysis}

The basic CFD software used to study the tank sloshing phenomenon is the commercially available CFD-ACE+ code, which was originally developed by CFD Research Corp., and is currently owned and distributed by ESI Group USA. Some modifications and improvements, which will be described in the following section, have been made to tackle the unique vehicle sloshing problem. CFD-ACE+ has a VOF (Volume of Fluid) module which is designed for applications involving two immiscible fluids. A single set of momentum and continuity equations is solved, but different property sets are defined for each fluid. The volume fraction of one phase (in this case LH2 or LOX) is tracked throughout the solution to determine which fluid occupies each computational cell at any given time. In cells containing both fluids, a special routine is used to locate the shape and location of the interface. When there exists a surface tension force, its effect is applied in a conservative form. For time dependent simulations, such as unsteady tank sloshing, a special high order algorithm is used to update the 
volume fraction in a cell from one time step to the next. A geometric reconstruction scheme for the interface representation is employed to track the interface. This unique algorithm is capable of capturing and maintaining a sharp interface between the phases for long time without introducing numerical diffusion. The time step size is determined by the local CFL number.

It should be emphasized that accurate modeling of interfacial flows requires high fidelity algorithm for the kinematics and dynamics of interfaces, especially when surface tension force is important. In CFD-ACE+, the Laplace-Young high-order nonlinear boundary conditions at the liquid surface are applied, and a conservative algorithm for surface tension force previously developed by Yang and Przekwas [1] was implemented. This conservative surface tension treatment has shown to give high numerical stability, and can accurately predict transport speed of the liquid front, which is very difficult to do with other solution techniques.

\subsection{Implementation of Body Forces due to Non-Inertial Frame of a Vehicle}

The Navier-Stokes equations for a CFD code are typically written in the inertial reference frame and are derived from Newton's law of motion in the form of:

$$
m \vec{a}=\vec{f}
$$

This law claims that the only way an object can change its velocity is if a non-zero net force is applied to the object. During the Crew Launch Vehicle (CLV) ascent, the Upper Stage (US) propellant tanks are in a non-inertial frame, in that the tanks are travelling along a curved path, and also speeding up or slowing down, and are subjected to continuous maneuvering including roll, pitch and yaw. In the non-inertial frame, Newton's laws of motion of equation (1) will no longer hold, and there arise fictitious forces. This section will derive the extra fictitious forces in the non-inertial frame for the N-S equations.

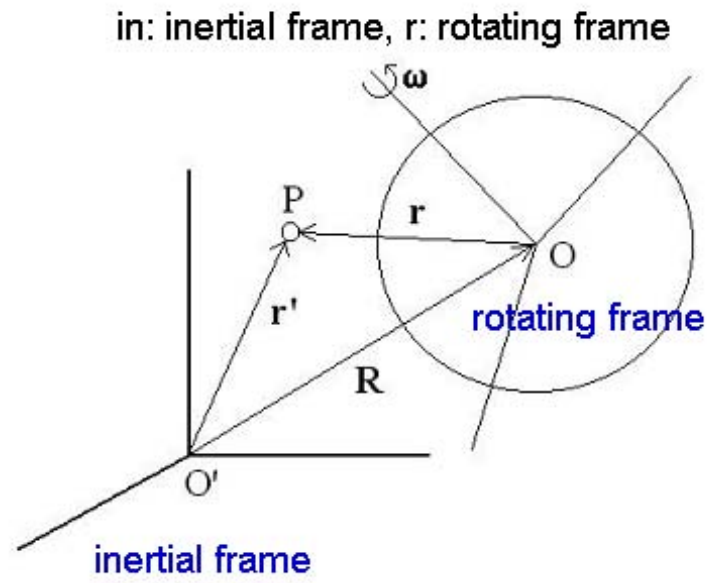

Figure 1. Inertial frame and non-inertial frame (rotating frame)

In the non-inertial frame, any vector A has a relation of:

$$
\left(\frac{d \vec{A}}{d t}\right)_{i n}=\left(\frac{d \vec{A}}{d t}\right)_{r}+\omega \times \vec{A}
$$


The subscript "in" refers to the inertial frame, and " $\mathrm{r}$ ” refers to rotating frame (or non-inertial frame). As shown in Figure 1,

$$
\text { let }: \vec{r}^{\prime}=\vec{R}+\vec{r}
$$

Then

$$
\left(\frac{d \vec{r}^{\prime}}{d t}\right)_{i n}=\left(\frac{d \vec{R}}{d t}\right)_{i n}+\left(\frac{d \vec{r}}{d t}\right)_{i n}=\vec{v}_{c g}+\vec{v}_{r}+\vec{\omega} \times \vec{r}
$$

Where, $v_{\text {cg }}$ denotes the translational velocity of the non-inertial frame origin (as observed in the fixed frame), $v_{r}$ is the velocity in the non-inertial frame relative to the origin of the frame. Now,

$$
\begin{aligned}
& \text { let }: \vec{a}_{i n}=\frac{d}{d t}\left(\frac{d \vec{r}^{\prime}}{d t}\right)_{i n}, \vec{a}_{r}=\left(\frac{d \vec{v}_{r}}{d t}\right)_{r} \text {, then } \\
& \vec{a}_{i n}=\left(\frac{d \vec{v}_{c g}}{d t}\right)_{i n}+\left(\frac{d \vec{v}_{r}}{d t}\right)_{i n}+\left(\frac{d \vec{\omega}}{d t}\right)_{i n} \times \vec{r}+\vec{\omega} \times\left(\frac{d \vec{r}}{d t}\right)_{i n} \\
& =\vec{a}_{c g}+\left(\vec{a}_{r}+\vec{\omega} \times \vec{v}_{r}\right)+\dot{\vec{\omega}} \times \vec{r}+\vec{\omega} \times\left(\vec{v}_{r}+\vec{\omega} \times \vec{r}\right) \\
& =\vec{a}_{c g}+\vec{a}_{r}+2 \vec{\omega} \times \vec{v}_{r}+\dot{\vec{\omega}} \times \vec{r}+\vec{\omega} \times(\vec{\omega} \times \vec{r})
\end{aligned}
$$

It is interesting to note that:

$\vec{a}_{c g}$ : acceleration of vehicle center of gravity (CG)

$\vec{a}_{r}$ : acceleration of fluid particles in the non-inertial frame

$2 \vec{\omega} \times \vec{v}_{r}$ : Coriolis acceleration

$\dot{\vec{\omega}} \times \vec{r}$ : angular acceleration

$\vec{\omega} \times(\vec{\omega} \times \vec{r})$ : centrifugal acceleration

With the above expressions, we can now write the Newton's Law as:

$$
\begin{array}{ll} 
& m \vec{a}_{i n}=\vec{f} \\
\text { or: } \quad & m\left(\vec{a}_{c g}+\vec{a}_{r}+2 \vec{\omega} \times \vec{v}_{r}+\dot{\vec{\omega}} \times \vec{r}+\vec{\omega} \times(\vec{\omega} \times \vec{r})=\vec{f}\right. \\
& m \vec{a}_{r}=\vec{f}-m \vec{a}_{c g}-m \dot{\vec{\omega}} \times \vec{r}-2 m \vec{\omega} \times \vec{v}_{r}-m \vec{\omega} \times(\vec{\omega} \times \vec{r}) \\
& m \vec{a}_{r}=\vec{f}+\vec{f}_{\text {user }}
\end{array}
$$

Here $\mathrm{f}_{\text {user }}$ assembles the fictitious body forces due to vehicle acceleration and maneuvering, and they are the terms to be added into an existing CFD code. Specifically:

$$
\begin{aligned}
& \left(f_{\text {user }}\right)_{x}=-m\left(a_{c g}\right)_{x}-m\left(\dot{\omega}_{y} r_{z}-\dot{\omega}_{z} r_{y}\right)-2 m\left(\omega_{y} v_{r z}-\omega_{z} v_{r y}\right)-m\left[-\left(\omega_{y}{ }^{2}+\omega_{z}{ }^{2}\right) r_{x}{ }^{2}+\omega_{x} \omega_{y} r_{y}+\omega_{x} \omega_{z} r_{z}\right] \\
& \left(f_{\text {user }}\right)_{y}=-m\left(a_{c g}\right)_{y}-m\left(\dot{\omega}_{z} r_{x}-\dot{\omega}_{x} r_{z}\right)-2 m\left(\omega_{z} v_{r x}-\omega_{x} v_{r z}\right)-m\left[-\left(\omega_{z}{ }^{2}+\omega_{x}{ }^{2}\right) r_{y}{ }^{2}+\omega_{y} \omega_{z} r_{z}+\omega_{y} \omega_{x} r_{x}\right] \\
& \left(f_{\text {user }}\right)_{z}=-m\left(a_{c g}\right)_{z}-m\left(\dot{\omega}_{x} r_{y}-\dot{\omega}_{y} r_{x}\right)-2 m\left(\omega_{x} v_{r y}-\omega_{y} v_{r x}\right)-m\left[-\left(\omega_{x}{ }^{2}+\omega_{y}{ }^{2}\right) r_{z}{ }^{2}+\omega_{z} \omega_{x} r_{x}+\omega_{z} \omega_{y} r_{y}\right]
\end{aligned}
$$


The above derived body forces due to non-inertial frame have been implemented into a user subroutine, which is then linked to the commercial CFD software, CFD-ACE+.

\section{VALIDATION STUDIES}

Sloshing is a wave phenomenon, and is driven by gravitational forces, surface tension, and surface kinematics. For LH2 and LOX propellant tanks, one can typically observe two types of surface sloshing waves: the first natural mode and the second natural mode. This is illustrated in Figure 3 for the LH2 tank and in Figure 4 for the LOX tank.
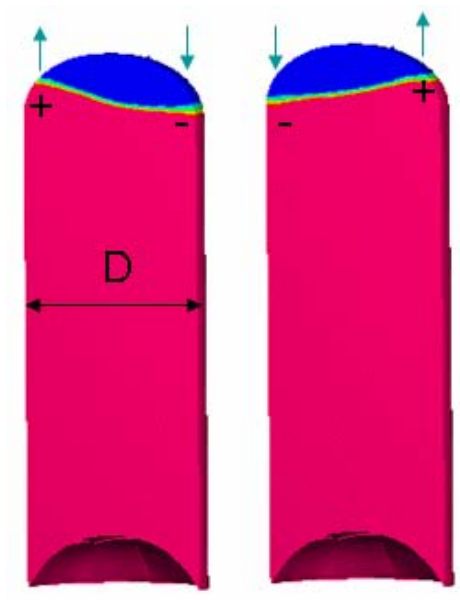

First Mode, $\lambda=2 \mathrm{D}$
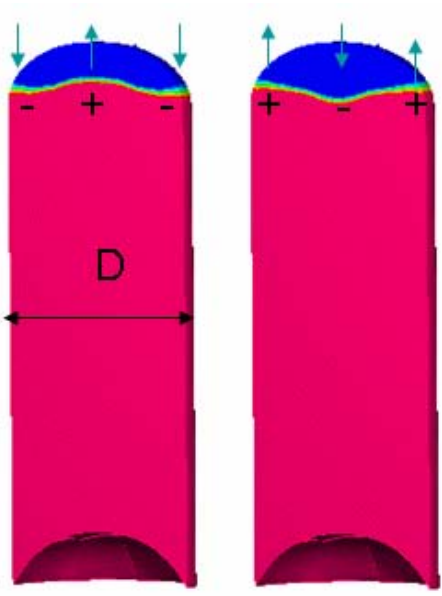

Second Mode, $\lambda=D$

Figure 3 The First Two Natural Modes Of LH2 Tank. D: Diameter Of The Tank, $\lambda$ : Wavelength, and g: gravitational vector.

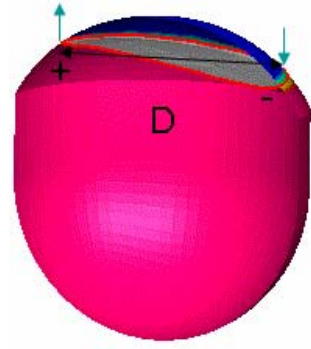

First Mode, $\lambda=2 \mathrm{D}$

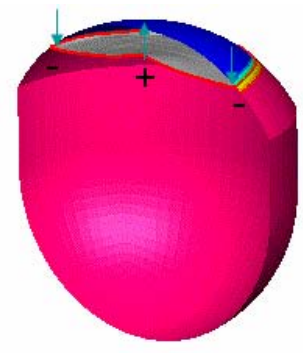

Second Mode, $\lambda=\mathrm{D}$

Figure 4 The First Two Natural Modes Of LOX Tank.

$D$ : Diameter Of The Tank, $\lambda$ : Wavelength.

These two modes are standing modes and have a direct relationship with the diameter of the tank. The first mode is symmetric with respect to the central plane, while the second mode is axisymmetric. Typically, in flight, the first mode is the dominant mode. It is important to predict the natural frequency, the participating sloshing mass, the damping, the mass center of these modes.

During the 1960's when CFD technology was in its infancy, the sloshing dynamics of liquid tank was studied either analytically or experimentally. In the analytical approach, assumptions have been made to arrive the closed form solutions. While in the experimental approach, only subscale models are tested and measured. The full scale sloshing mechanical parameters have to 
be extrapolated from the sub-scale model. To build confidence in using CFD technology to extract and analyze the sloshing dynamics, this paper will describe our effort to validate the CFD model against both analytical derivations and experimental measurements.

\subsection{Validation Against Analytical Solutions}

Southwest Research Institute has been involved in sloshing research since before the start of the US space program. Dr. Norm Abamson, then the leader of the sloshing group has organized and published a monograph [2] which contained very comprehensive knowledge on both experimental and analytical results of sloshing and related subjects. The analytical solutions were derived for simple geometries such as rectangular boxes or a cylindrical tank with a flat bottom face. These analytical solutions have been currently used at MSFC to get mechanical parameters (mass, frequency, damping, mass center) for the preliminary design and for sloshing model input into GN\&C’s MAVERIC model. In reality, the analytical results are applicable only to the simple geometries such as cylindrical and spherical tanks. We will use the example of cylindrical tank to compare CFD predicted mechanical parameters with the analytical solutions.

Simulation Model. The computational model along with the computational grid is shown in Figure 5. Here a cylindrical tank with diameter $\mathrm{D}=3$ ” and height of $\mathrm{H}=11$ ” is modeled. The tank has no internal obstruction and all the walls are smooth. The top of the tank is open to ambient condition and is set up as fixed pressure boundary condition. All other boundaries are set as non-slip wall. There are a total of 80,000 cells, with 60 cells covering the whole tank diameter. This ensures that there will be at least 120 cells to resolve the first natural mode and 60 cells to resolve the second mode. Butterfly grid is used to avoid the singularity at the tank center line, and to give better uniformity in the grid size. To determine the natural frequency of the tank slosh, an initial inclined surface is specified with a slope of 5.7 deg, as shown in Figure 5. A typical time dependent mass center coordinate of the liquid mass inside the tank, computed by integrating the Volume of the Fluid value in each cell, is shown in Figure 6 .
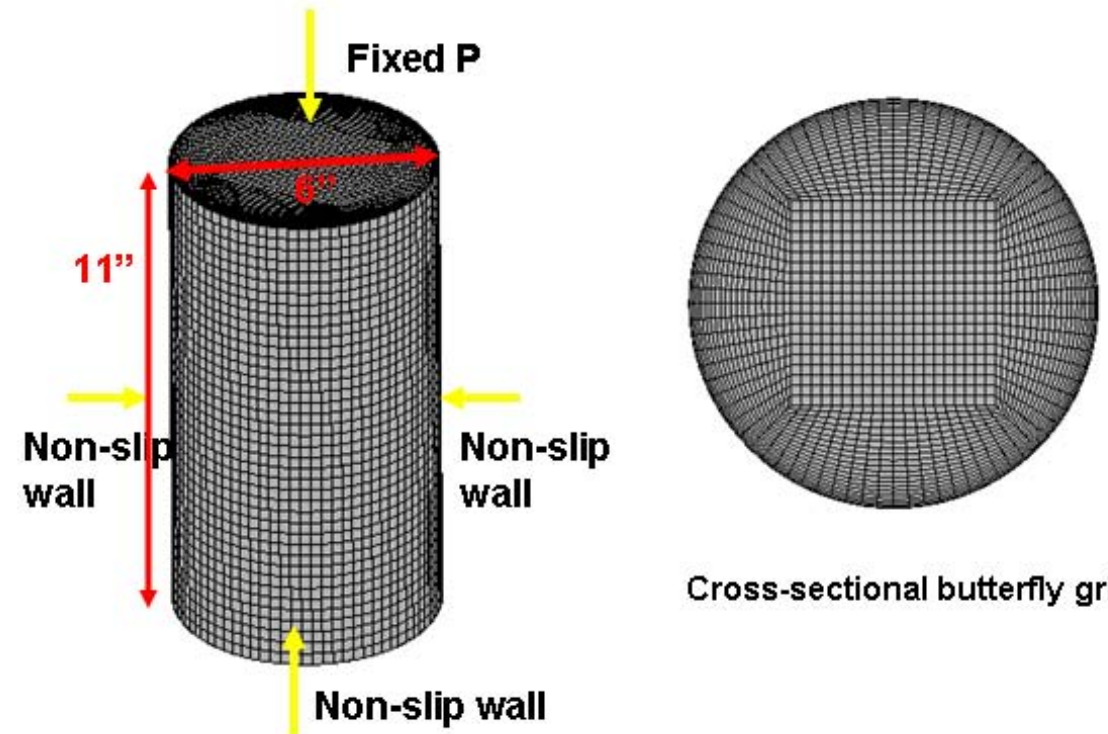

Cross-sectional butterfly grid

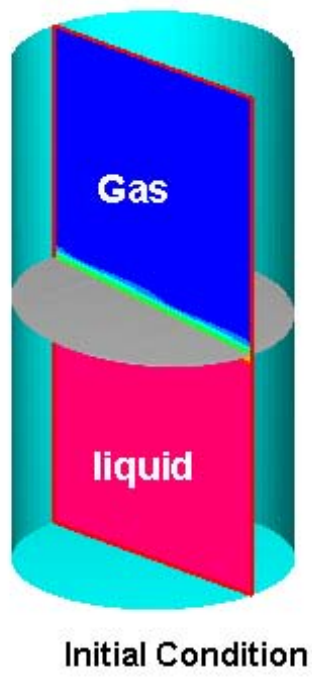

Figure 5. CFD model of a cylinder tank sloshing for comparison with analytical solutions. 


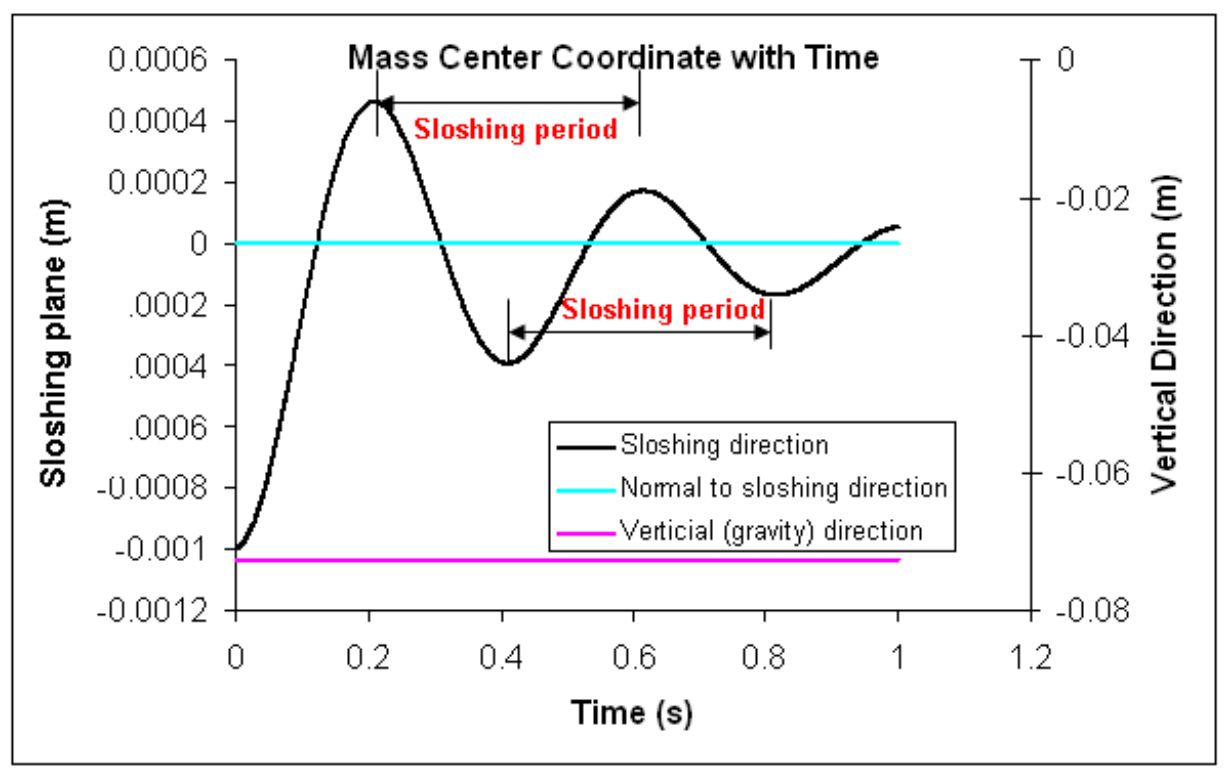

Figure 6. The center coordinates of the total liquid mass in the tank

As seen from Figure 6, the vertical coordinate of the mass center is essentially constant, implying that the sloshing mass center does not move in the vertical direction, which is due to the symmetrical properties of surface wave. One may also notice an almost zero value of the mass center in the direction normal to the sloshing plane. This indicates that sloshing is essentially two dimensional.

\section{Extraction of Natural Frequency}

From Figure 6 one can see that the sloshing has a rather constant period, which makes it possible to extract the natural frequency of the tank sloshing from the above data. Several simulations were made at different heights of the liquid level, and they are summarized in Table 1. For the same configuration, one can use the analytical solution to predict the natural frequency as [3], which is also presented in Table 1:

$$
f_{1}=\frac{1}{2 \pi} \sqrt{\frac{1.841 g}{R} \tanh \left(\frac{1.841 h}{R}\right)}
$$

Table 1. Sloshing Frequency Comparison for a Cylinder Tank in Water, R=3"

\begin{tabular}{|c|c|c|c|c|}
\hline Liquid Level & $\mathrm{h} / \mathrm{R}=2.0$ & $\mathrm{~h} / \mathrm{R}=1.0$ & $\mathrm{~h} / \mathrm{R}=0.5$ & $\mathrm{~h} / \mathrm{R}=0.25$ \\
\hline Analytical & $2.4523 \mathrm{~Hz}$ & $2.3880 \mathrm{~Hz}$ & $2.0867 \mathrm{~Hz}$ & $1.6058 \mathrm{~Hz}$ \\
\hline Present CFD & $2.4539 \mathrm{~Hz}$ & $2.3753 \mathrm{~Hz}$ & $2.0920 \mathrm{~Hz}$ & $1.6155 \mathrm{~Hz}$ \\
\hline Error & $0.06 \%$ & $0.53 \%$ & $0.56 \%$ & $0.97 \%$ \\
\hline
\end{tabular}

Table 1 also shows the analytical solution, the CFD predictions and the difference presented in percentage error. One can clearly see that the CFD prediction gives excellent agreement with the analytical solution for all the liquid levels. This builds up the confidence of the CFD analysis. 
Extraction of Slosh Mass and Slosh Mass Center from CFD Solution

In addition to extracting the sloshing natural frequency, one can also extract sloshing mass and the sloshing mass center. The modeling procedure is to apply a periodic side load to force the liquid in the tank into a period motion at the forcing frequency. From the mass center amplitude, one can determine the equivalent sloshing mass of the system. The procedure and sample results are illustrated in Figure 7. Table 2 shows the comparison of the CFD prediction and analytical solution for the slosh mass ratio. Again, one can clearly see the results are in excellent agreement.

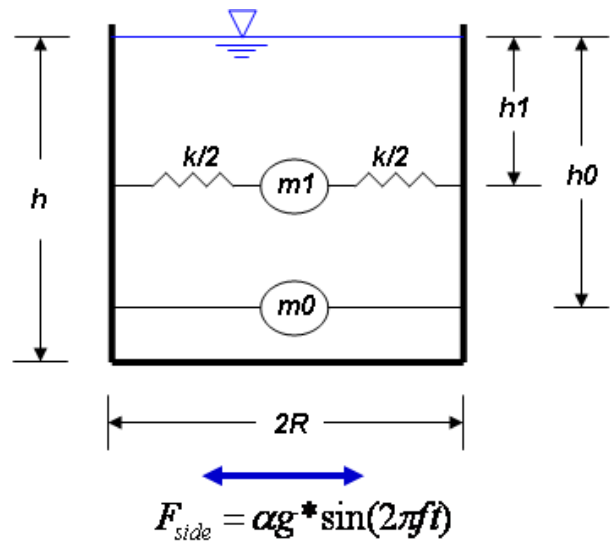

Extraction of Slosh Mass Procedure

1. Apply an oscillatory side force to the tank at frequency $f_{0}$, and amplitude $\alpha g$, other than natural frequency $f_{n}$

$$
F_{\text {side }}=\alpha \mathrm{g} * \sin \left(2 \pi f_{0} t\right)
$$

2. Once the mass center location reaches periodic state with maximum amplitude $\mathrm{x}_{\max }$, determine the mass ratio from:

$$
\frac{\boldsymbol{m}_{1}}{\boldsymbol{m}}=\frac{\boldsymbol{x}_{\max }}{\alpha g}\left[\left(2 \pi f_{n}\right)^{2}-\left(2 \pi f_{0}\right)^{2}\right]
$$
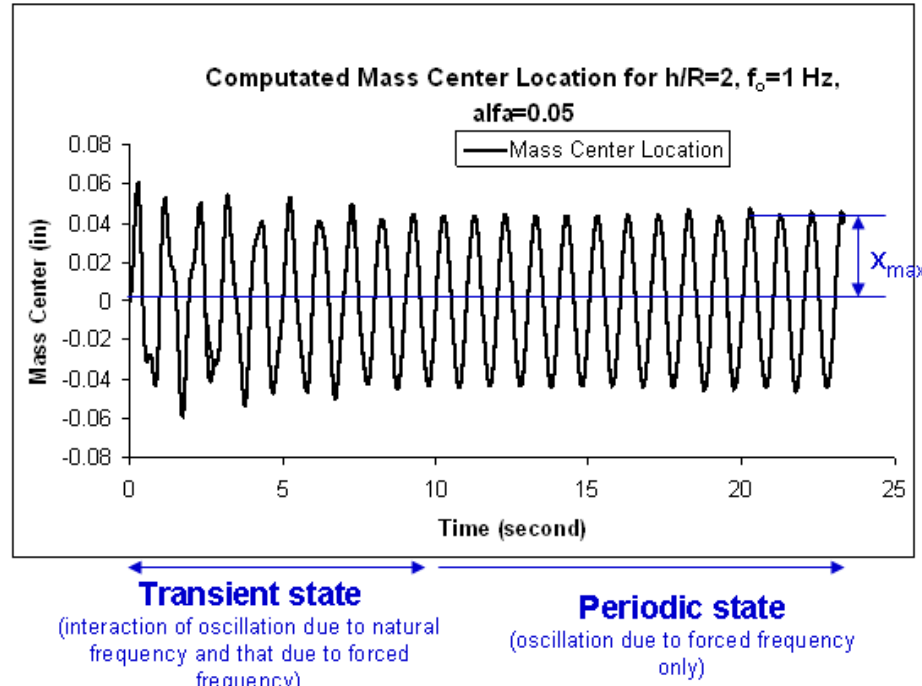

Sample Mass Center History

Figure 7. Procedure to extract sloshing mass.

Table 2. Sloshing Mass Ratio (m1/m) Comparison for a Cylinder Tank in Water, R=3”

\begin{tabular}{|c|c|c|c|c|}
\hline Liquid Level & $\mathrm{h} / \mathrm{R}=2.0$ & $\mathrm{~h} / \mathrm{R}=1.0$ & $\mathrm{~h} / \mathrm{R}=0.5$ & $\mathrm{~h} / \mathrm{R}=0.25$ \\
\hline Analytical & 0.22735 & 0.43236 & 0.66028 & 0.78252 \\
\hline Present CFD & 0.22858 & 0.43380 & 0.66402 & 0.77950 \\
\hline Error & $0.54 \%$ & $0.33 \%$ & $0.56 \%$ & $0.39 \%$ \\
\hline
\end{tabular}

\subsection{Comparison with Experimental Data}


The model is a 1/3.75 scale oblate spherical Centaur LOX tank, and experiments were carried out during the 1960's by Sumner et al. [3]. As shown in Figure 8, excellent agreement was found between the present CFD prediction and the experimental measurement.

1/3.75 scale model of oblate spherical Centaur LOX tank
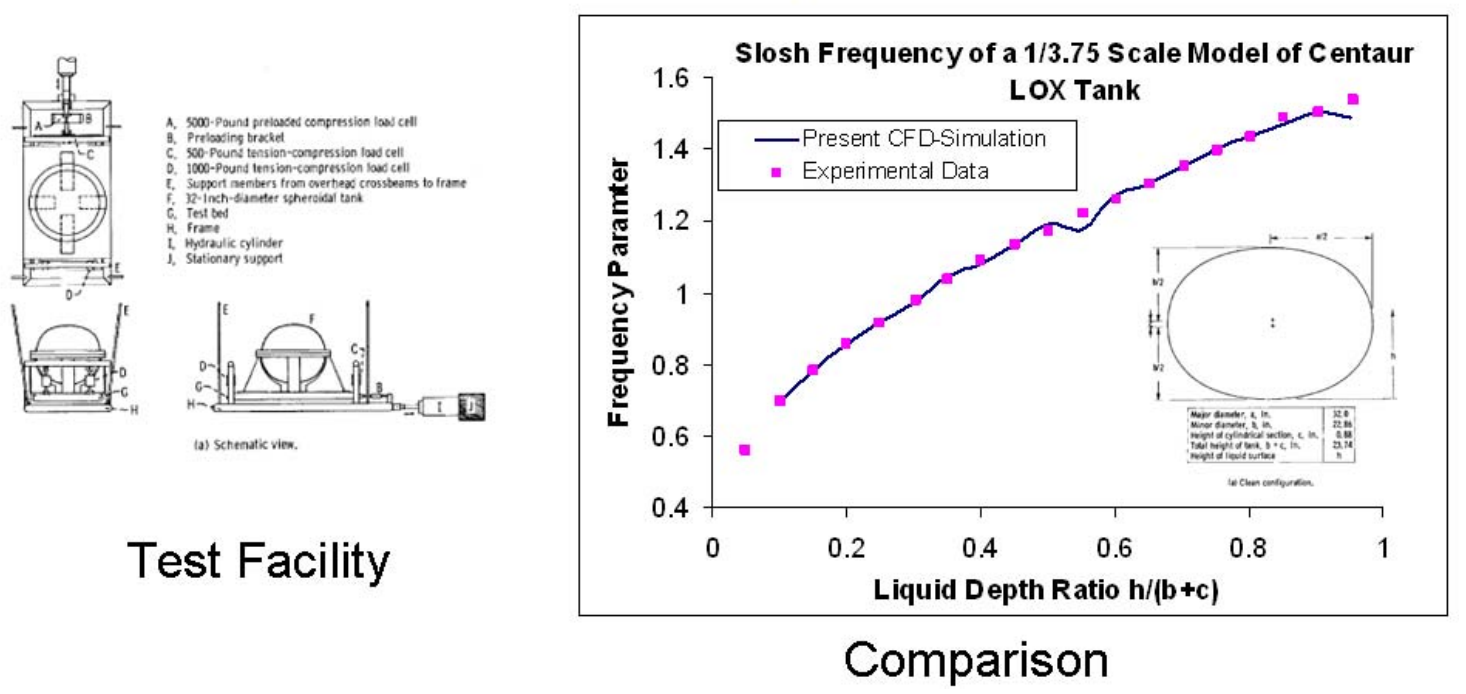

Figure 8. Comparison of CFD extracted frequency vs. experiments for 1/3.75 scale model of Centaur LOX Tank.

\section{3. $\quad$ Advantage in Using CFD Predicted Mechanical Model}

The previous section has clearly illustrated the soundness of the CFD approach in modeling detailed fluid dynamics of tank sloshing and with excellent accuracy in extracting mechanical properties for different configurations. This is especially applicable to the future design of propellant tanks, as there is currently no experimental data available for the same size and configuration. Table 3 lists a few points that CFD can help to improve the extraction of damped spring mass mechanical parameters. 


\begin{tabular}{|c|c|c|c|}
\hline & $\begin{array}{l}\text { Assumptions } \\
\text { in Analysis }\end{array}$ & $\begin{array}{l}\text { Limitations of the } \\
\text { Assumptions }\end{array}$ & $\begin{array}{l}\text { Advantages of CFD } \\
\text { Modeling }\end{array}$ \\
\hline Tank Geometry & $\begin{array}{l}\bullet \text { Flat bottom } \\
\text { cylinders }\end{array}$ & $\begin{array}{l}\text { - Good for LH2 tank } \\
\text { - Questionable for } \\
\text { LOX tank }\end{array}$ & $\begin{array}{l}\text { - Realistically true tank } \\
\text { geometry for Ares I tanks }\end{array}$ \\
\hline Liquid Height & $\begin{array}{l}\text { - Limiting shallow } \\
\text { tank } \\
\text { • Limiting deep tank }\end{array}$ & $\begin{array}{l}\text { - Good for LH2 tank } \\
\text { during the first stage. } \\
\text { - Not valid for LOX } \\
\text { tank }\end{array}$ & $\begin{array}{l}\text { - No limitation } \\
\text { - Applicable to all liquid } \\
\text { heights during the first and } \\
\text { second stages }\end{array}$ \\
\hline $\begin{array}{l}\text { Sloshing } \\
\text { Amplitude }\end{array}$ & - Linea & $\begin{array}{l}\text { - Large amplitude slosh } \\
\text { could be encountered } \\
\text { - Applicability to large } \\
\text { amplitude slosh during } \\
\text { flight is unknown. }\end{array}$ & $\begin{array}{l}\text { - Able to simulate the } \\
\text { whole trajectory with small } \\
\text { and large amplitudes. } \\
\text { b Can shed light on the } \\
\text { limitations of linear } \\
\text { theory. }\end{array}$ \\
\hline Ullage Gas & $\begin{array}{l}\text { - No ullage gas or } \\
\text { gas density is zero } \\
\text { - No effect of ullage } \\
\text { gas on the surface } \\
\text { dynamics }\end{array}$ & $\begin{array}{l}\bullet \text { Good for water and } \\
\text { air. } \\
\text { - Not good for } \\
\text { cryogenic fluids with } \\
\text { higher density ratio of } \\
\text { gas to liquid. }\end{array}$ & $\begin{array}{l}\text { Able to correct any error } \\
\text { from the theory using the } \\
\text { realistic properties, } \\
\text { especially for the first } \\
\text { sloshing frequency. }\end{array}$ \\
\hline $\begin{array}{l}\text { Gravity (Body) } \\
\text { Force }\end{array}$ & $\begin{array}{l}\text { - Constant } \\
\text { - Neglected dynamic } \\
\text { effect }\end{array}$ & $\begin{array}{l}-\mathrm{g} \text { is a function of time } \\
\text { during flight. }\end{array}$ & $\begin{array}{l}\text { • Dynamic effect are taken } \\
\text { into account. } \\
\text { • Can handle variable g. }\end{array}$ \\
\hline
\end{tabular}

More detailed comparison with experimental data will be reported in the full paper.

\section{REFERENCES:}

1. H. Q. Yang, and A. J. Przekwas, "Computational Modeling of Microfluid Devices with Free Surface Liquid Handling”, Technical Proceeding of the 1998 International Conference on Modeling and Simulation of Microsystems, MSM 98, pp. 498-505, 1998.

2. H. N. Abramson, "The Dynamic Behavior of Liquids in Moving Containers", NASA SP106, 1967.

3. I. E. Sumner, A. J. Stofan and D. J. Shramo, "Experimental Sloshing Characteristics and a Mechanical Analogy of Liquid Sloshing in a Scale-Model Centaur Liquid-Oxygen Tank”, NASA TM-X-999, August, 1964. 


\title{
Propellant Sloshing Parameter Extraction from CFD Analysis
}

\author{
H. Q. Yang ${ }^{1}$ \\ CFD Research Corp. \\ and \\ John Peupeot ${ }^{2}$ \\ ER42 NASA MSFC
}

\begin{abstract}
Propellant slosh is a potential source of disturbance critical to the stability of space vehicle. The sloshing dynamics is typically represented by a mechanical model of spring mass damper. This mechanical model is then included in the equation of motion of the entire vehicle for Guidance, Navigation and Control analysis. The typical parameters required by the mechanical model include natural frequency of the sloshing, sloshing mass, sloshing mass center coordinates, and critical damping coefficient. During the 1960's US space program, these parameters were either computed from analytical solution for simple geometry or by experimental testing for the sub-scaled configurations. The purpose of this work is to demonstrate the soundness of a CFD approach in modeling the detailed fluid dynamics of tank sloshing and the excellent accuracy in extracting mechanical properties for different tank configurations and at different fill levels. The validation studies included straight cylinder against analytical solution, and sub-scaled Centaur LOX and LH2 tanks with and without baffles against experimental results. This effort shows that CFD technology can provide accurate mechanical parameters for any tank configuration, and is especially valuable to the future design of propellant tanks, as there is no previous experimental data available for the same size and configuration.
\end{abstract}

\section{Introduction}

$\mathrm{P}$ ropellant sloshing is a potential source of disturbance critical to the stability of launch vehicles. The driving sloshing forces of the propellant mass come from lateral disturbance, oscillatory thrust force, or angular rotation of the vehicle during the powered phase of the flight. As such, sloshing is expected to occur during vehicle taxi, takeoff, engine shut off, and flight maneuvers. Fluid motion produced during these operations can circulate subcooled liquid fuel near the liquid vapor interface resulting in increased condensation and corresponding pressure collapse. Conversely, liquid contact on hot tank walls caused by sloshing can result in rapid vaporization and subsequent rapid pressure rise. Understanding the dynamic behavior and the response of cryogenic propellants of Liquid Hydrogen (LH2) and Liquid Oxygen (LOX) tanks under spacecraft flight operations (such as engine restart, oscillatory thrust and oscillatory side forces) is extremely important during the vehicle design. In addition, the proper orientation of propellant is necessary for improved performance of the tank vent system, to ensure sufficient liquid propellant for engine firing and proper thermal conditions in feed lines prior to ignition, and to minimize vehicle disturbances and avoid excessive demands on the attitude controls.

Sloshing is a wave phenomenon, and is driven by gravitational forces, surface tension, and surface kinematics. To take all the detailed dynamics into account in a vehicle dynamic modeling is very time consuming. On the other hand, a complete dynamic-stability analysis of the vehicle must include the forces produced by the liquid motion in partially filled propellant tanks. The sloshing dynamics is typically represented by a mechanical model of spring mass damper. This mechanical model is then included in the equation of motion of the entire vehicle for Guidance, Navigation and Control Analysis (GN\&C) analysis. The quantities that are input to the GN\&C analysis to consider the propellant sloshing are: natural frequency of the sloshing, sloshing mass, sloshing mass center coordinates, and critical damping coefficient. Many analytical solutions have been made to determine these sloshing

${ }^{1}$ Chief Scientist/Research, 215 Wynn Drive, Huntsville, AL 35805, and Senior AIAA Member.

${ }^{2}$ Aerospace Engineers, Fluid Dynamics Branch-ER42, George C. Marshall Space Slight Center, MSFC, AL 35812.

1

American Institute of Aeronautics and Astronautics 
mechanical parameters, but their accuracy and applicability to the real configuration are not well known for vehicle specific flight tanks.

Using first principle based Newtonian fluid dynamics, NASA Marshall Space Flight Center has developed a high fidelity Computational Fluid Dynamics (CFD) model for simulation and analysis of sloshing phenomenon. This model takes into account all the essential physics of the liquid-gas interface, effect of gravitational forces, surface tension and surface kinematics, to model tank slosh using the acceleration forces provided by GN\&C. The objective of this study is to validate CFD extracted mechanical parameters (such as sloshing mass, sloshing frequency, and sloshing mass center) against theoretical solutions derived in 1960's for simple cylindrical tanks and against experimental data taken during the 1960's for more complex geometries.

\section{Analysis Modeling Approach}

\section{A. Fluid Dynamics Analysis}

The basic CFD software used to study the tank sloshing phenomenon is the commercial CFD-ACE+ code, which was originally developed by CFD Research Corp., and is currently owned and distributed by ESI Group USA. Some modification and improvement, which will be described in the following section, have been made to tackle the present unique vehicle sloshing problem. CFD-ACE+ has a VOF (Volume of Fluid) module which is designed for applications involving two immiscible fluids. A single set of momentum and continuity equations is solved, but different property sets are defined for each fluid. The volume fraction of one phase (in this case LH2 or LOX) is tracked throughout the solution to determine which fluid occupies each computational cell at any given time. In cells containing both fluids, a special routine is used to locate the shape and location of the interface. When there exists a surface tension force, its effect is applied in a conservative form. For time dependent simulations such as the present tank sloshing, a special high order algorithm is used to update the volume fraction in a cell from one time step to the next. The geometric reconstruction scheme for the interface representation is employed to track the interface. This unique algorithm is capable of capturing and maintaining a sharp interface between the phases for long time without introducing numerical diffusion. The time step size is determined by the local CFL number.

It should be emphasized that accurate modeling of interfacial flows requires a high fidelity algorithm for the kinematics and dynamics of interfaces, especially when surface tension forces are important. In CFD-ACE+, the Laplace-Young high-order nonlinear boundary conditions at the liquid surface are applied, and a conservative algorithm for surface tension force previously developed by Yang and Przekwas [1] was implemented. This conservative surface tension treatment has shown to give high numerical stability, and can accurately predict transport speed of liquid front, which is very difficult to do with other solution techniques.

\section{B. Implementation of Body Forces due to Non-Inertial Frame of a Vehicle}

The Navier-Stokes equations for a CFD code are typically written in the inertial reference frame and are derived from Newton's law of motion in the form of:

$$
m \vec{a}=\vec{f}
$$

This law claims that the only way an object can change its velocity is if a non-zero net force is applied to the object. During the Crew Launch Vehicle (CLV) ascent, the Upper Stage (US) propellant tanks are in a non-inertial frame, in that the tanks are travelling along a curved path, and also speeding up or slowing down, and are subjected to continuous maneuvering of roll, pitch and yaw. In the non-inertial frame, Newton's laws of motion of equation (1) will no longer hold, and there arise fictitious forces. This section will derive the extra fictitious forces in the non-inertial frame for the N-S equations. 


\section{in: inertial frame, r: rotating frame}

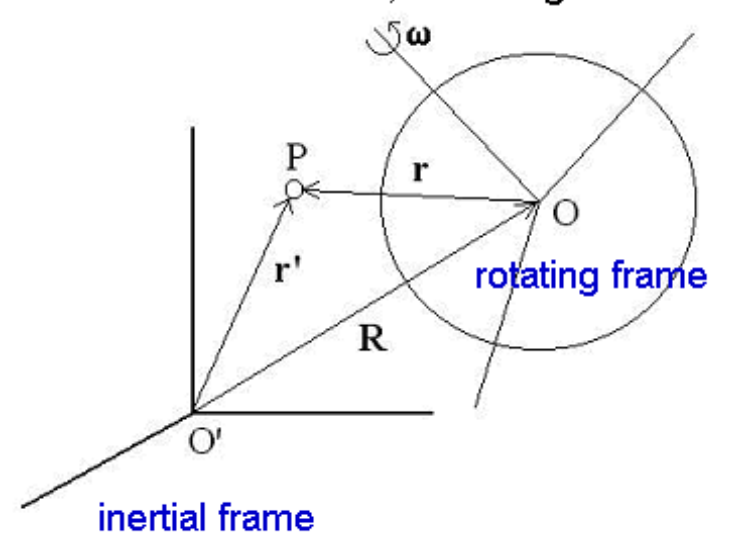

Figure 1. Inertial frame and non-inertial frame (rotating frame)

In the non-inertial frame, any vector A has a relation of:

$$
\left(\frac{d \vec{A}}{d t}\right)_{i n}=\left(\frac{d \vec{A}}{d t}\right)_{r}+\omega \times \vec{A}
$$

With subscript "in" refers to the inertial frame, and " $r$ " refers to rotating frame (or non-inertial frame). As shown in Figure 1,

Then

$$
\text { let }: \vec{r}^{\prime}=\vec{R}+\vec{r}
$$

$$
\left(\frac{d \vec{r}^{\prime}}{d t}\right)_{i n}=\left(\frac{d \vec{R}}{d t}\right)_{i n}+\left(\frac{d \vec{r}}{d t}\right)_{i n}=\vec{v}_{c g}+\vec{v}_{r}+\vec{\omega} \times \vec{r}
$$

Where, $\mathrm{v}_{\mathrm{cg}}$ denotes the translational velocity of the non-inertial frame origin (as observed in the fixed frame), $\mathrm{v}_{\mathrm{r}}$ is the velocity in the non-inertial frame relative to the origin of the frame. Now,

$$
\begin{aligned}
& \text { let }: \vec{a}_{i n}=\frac{d}{d t}\left(\frac{d \vec{r}^{\prime}}{d t}\right)_{i n}, \vec{a}_{r}=\left(\frac{d \vec{v}_{r}}{d t}\right)_{r}, \text { then } \\
& \vec{a}_{i n}=\left(\frac{d \vec{v}_{c g}}{d t}\right)_{i n}+\left(\frac{d \vec{v}_{r}}{d t}\right)_{i n}+\left(\frac{d \vec{\omega}}{d t}\right)_{i n} \times \vec{r}+\vec{\omega} \times\left(\frac{d \vec{r}}{d t}\right)_{i n} \\
& =\vec{a}_{c g}+\left(\vec{a}_{r}+\vec{\omega} \times \vec{v}_{r}\right)+\dot{\vec{\omega}} \times \vec{r}+\vec{\omega} \times\left(\vec{v}_{r}+\vec{\omega} \times \vec{r}\right) \\
& =\vec{a}_{c g}+\vec{a}_{r}+2 \vec{\omega} \times \vec{v}_{r}+\dot{\vec{\omega}} \times \vec{r}+\vec{\omega} \times(\vec{\omega} \times \vec{r})
\end{aligned}
$$

It is interesting to note that:

$\vec{a}_{c g}:$ acceleration of vehicle center of gravity (CG)

$\vec{a}_{r}:$ acceleration of fluid particles in the non-inertial frame

$2 \vec{\omega} \times \vec{v}_{r}:$ Coriolis acceleration

$\dot{\vec{\omega}} \times \vec{r}$ : angular acceleration

$\vec{\omega} \times(\vec{\omega} \times \vec{r})$ : centrifugal acceleration

With the above expressions, we can now write the Newton's Law as: 
or:

$$
m \vec{a}_{i n}=\vec{f}
$$

Finally

$$
m\left(\vec{a}_{c g}+\vec{a}_{r}+2 \vec{\omega} \times \vec{v}_{r}+\dot{\vec{\omega}} \times \vec{r}+\vec{\omega} \times(\vec{\omega} \times \vec{r})=\vec{f}\right.
$$

$$
m \vec{a}_{r}=\vec{f}-m \vec{a}_{c g}-m \dot{\vec{\omega}} \times \vec{r}-2 m \vec{\omega} \times \vec{v}_{r}-m \vec{\omega} \times(\vec{\omega} \times \vec{r})
$$

$$
m \vec{a}_{r}=\vec{f}+\vec{f}_{\text {user }}
$$

Here $f_{\text {user }}$ assembles the fictitious body forces due to vehicle acceleration and maneuvering, and they are the terms to be added into an existing CFD code. Specifically:

$$
\begin{aligned}
& \left(f_{u s e r}\right)_{x}=-m\left(a_{c g}\right)_{x}-m\left(\dot{\omega}_{y} r_{z}-\dot{\omega}_{z} r_{y}\right)-2 m\left(\omega_{y} v_{r z}-\omega_{z} v_{r y}\right)-m\left[-\left(\omega_{y}{ }^{2}+\omega_{z}{ }^{2}\right) r_{x}+\omega_{x} \omega_{y} r_{y}+\omega_{x} \omega_{z} r_{z}\right] \\
& \left(f_{u s e r}\right)_{y}=-m\left(a_{c g}\right)_{y}-m\left(\dot{\omega}_{z} r_{x}-\dot{\omega}_{x} r_{z}\right)-2 m\left(\omega_{z} v_{r x}-\omega_{x} v_{r z}\right)-m\left[-\left(\omega_{z}{ }^{2}+\omega_{x}{ }^{2}\right) r_{y}+\omega_{y} \omega_{z} r_{z}+\omega_{y} \omega_{x} r_{x}\right] \\
& \left(f_{u s e r}\right)_{z}=-m\left(a_{c g}\right)_{z}-m\left(\dot{\omega}_{x} r_{y}-\dot{\omega}_{y} r_{x}\right)-2 m\left(\omega_{x} v_{r y}-\omega_{y} v_{r x}\right)-m\left[-\left(\omega_{x}{ }^{2}+\omega_{y}{ }^{2}\right) r_{z}+\omega_{z} \omega_{x} r_{x}+\omega_{z} \omega_{y} r_{y}\right]
\end{aligned}
$$

The above derived body forces due to non-inertial frame have been implemented into a user subroutine, which is then linked to the commercial CFD software, CFD-ACE+.

\section{Validation Studies}

Sloshing is a wave phenomenon, and is driven by gravitational forces, surface tension, and surface kinematics. For LH2 and LOX propellant tanks, one can typically observe two types of surface sloshing waves: the first natural mode and the second natural mode. This is illustrated in Figure 2 for the LH2 tank and in Figure 3 for the LOX tank.

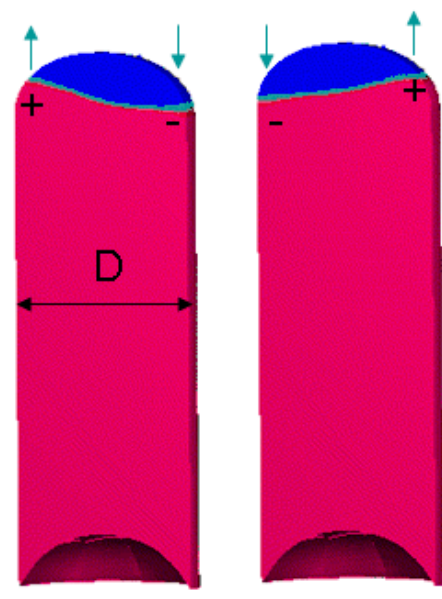

First Mode, $\lambda=2 \mathrm{D}$

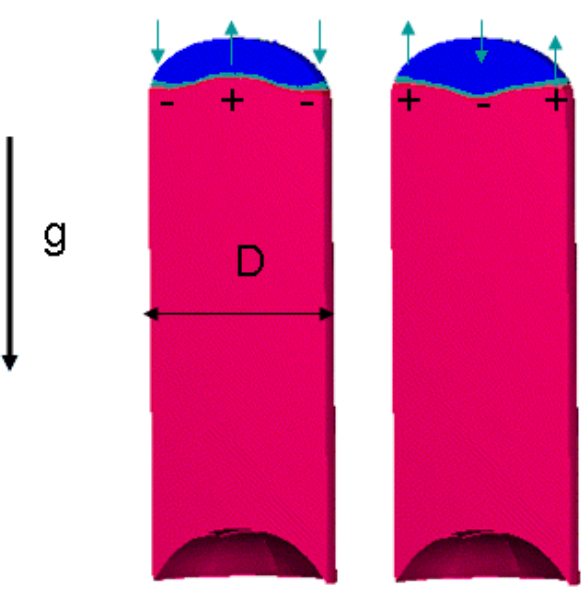

Second Mode, $\lambda=\mathrm{D}$

Figure 2. The First Two Natural Modes Of LH2 Tank. D: Diameter Of The Tank, $\lambda$ : Wavelength, and g: gravitational vector. 

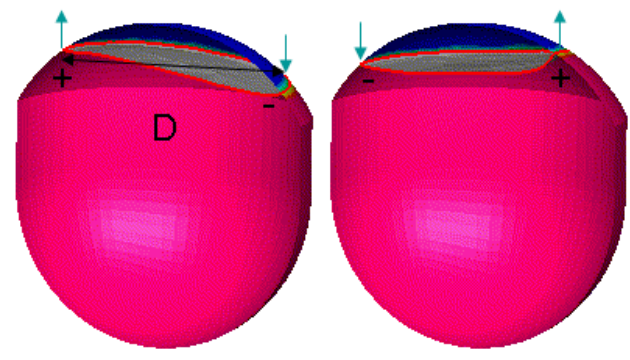

First Mode, $\lambda=2 D$
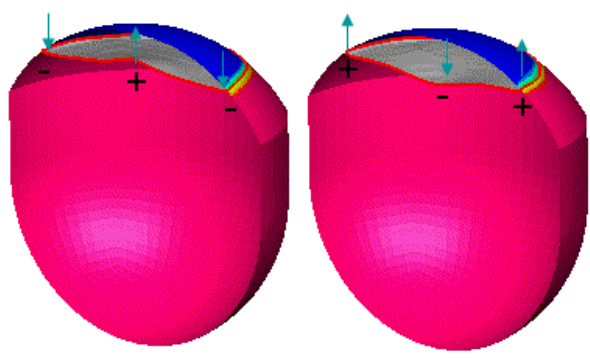

Second Mode, $\lambda=D$

Figure 3. The First Two Natural Modes Of LOX Tank. D: Diameter Of The Tank, $\lambda$ : Wavelength

These two modes are standing modes and have a direct relationship with the diameter of the tank. The first mode is symmetric with respect to the central plane, while the second mode is axisymmetric. In the typical application, the first mode is the dominant mode. It is important to predict the natural frequency, the participating sloshing mass, the damping, and the mass center of these modes.

During the 1960's when CFD technology was in its infancy, the sloshing dynamics of liquid tanks was studied either analytically or experimentally. In the analytical approach, assumptions have been made such that a closed form solution can be obtained. While in the experimental approach, only sub-scale models are tested and measured. The full scale sloshing mechanical parameters have to be extrapolated from the sub-scale model. To build confidence in using CFD technology for the extraction and analysis of sloshing dynamics, this paper will describe our effort in validating the CFD model against both analytical derivations and experimental measurements.

\section{A. Validation Against Drop Tower Test}

First, the CFD-ACE+ code was validated for the sloshing problem of cryogenic propellant during spacecraft flight operation. The ground experiments were carried out at Marshall Space Flight Center using a drop tower facility to obtain a controlled low gravity environment for duration of up to 4.3 seconds [2]. The test was conducted in six-inch diameter cylinders (see Figure 4) using petroleum Ether as the test fluid. The test geometry in a planar presentation is shown in Figure 4(b). Due to symmetry, only half of the tank model is considered, as shown in Figure 4(c).

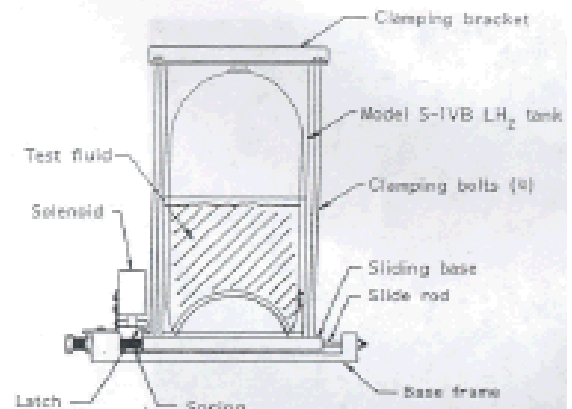

(a). Testing Equipment

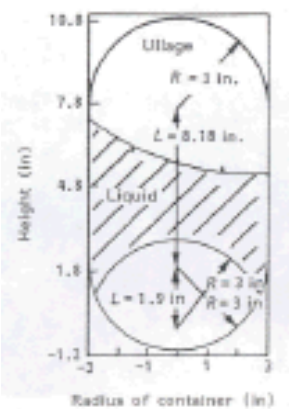

(b) Cross-section.

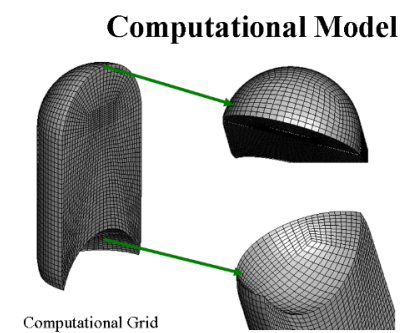

(c) 3D model and grid

\section{Figure 4. Experimental Package, Test Model and Simulation Model} 5 .

The initial conditions, physical properties, and profile of reduction in acceleration with time are listed in Figure 


\title{
Initial Conditions and Physical Properties
}

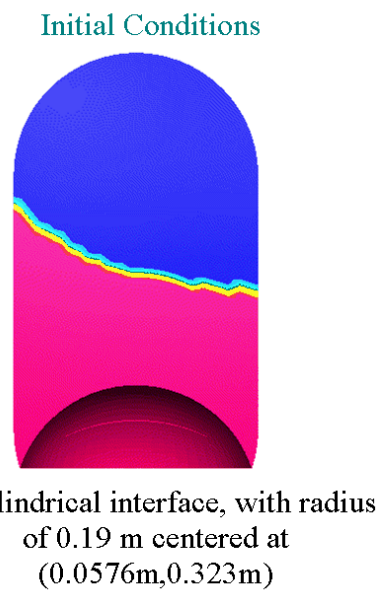

\author{
Physical Properties \\ Density $=618 \mathrm{~kg} / \mathrm{m}^{3}$ \\ Surface Tension $=0.0149 \mathrm{~N} / \mathrm{m}$ \\ Viscosity $=0.0002488 \mathrm{~kg} / \mathrm{m}-\mathrm{s}$ \\ Gravity Acceleration \\ $-9.8 \mathrm{~m} / \mathrm{s}^{2} \quad 0.0<\mathrm{t}<0.08 \mathrm{~s}$ \\ $-1.75 \mathrm{~m} / \mathrm{s}^{2} \quad 0.08 \mathrm{~s}<\mathrm{t}<0.09 \mathrm{~s}$ \\ $-1.06 \mathrm{~m} / \mathrm{s}^{2} \quad 0.09 \mathrm{~s}<\mathrm{t}<0.10 \mathrm{~s}$ \\ $-0.89 \mathrm{~m} / \mathrm{s}^{2} \quad 0.10 \mathrm{~s}<\mathrm{t}<0.20 \mathrm{~s}$ \\ $-0.51 \mathrm{~m} / \mathrm{s}^{2} \quad 0.20 \mathrm{~s}<\mathrm{t}<0.30 \mathrm{~s}$ \\ $-0.21 \mathrm{~m} / \mathrm{s}^{2} \quad 0.30 \mathrm{~s}<\mathrm{t}<0.356 \mathrm{~s}$ \\ $-0.10 \mathrm{~m} / \mathrm{s}^{2} \quad 0.356 \mathrm{~s}<\mathrm{t}<4.0 \mathrm{~s}$
} $(0.0576 \mathrm{~m}, 0.323 \mathrm{~m})$

\section{Figure 5. Initial Conditions and Physical Properties of Modeled Drop Tower Test}

The computational results from the present CFD-ACE+ simulation, an experimental photograph, and a previous simulation using FLOW-3D [3] software are shown in Figure 6 at several selected time instances. One can see good agreement between the flow fields from CFD-ACE+ simulation and those of FLOW-3D. The results also match well with the experimental data over the entire test duration.

\section{B. Validation against 2D Analytical Solution of Gravitational Wave}

In this section, an analytical solution is applied at the limiting condition of a $2 \mathrm{D}$ planar surface to validate the natural frequencies from the CFD simulations. Let us consider the two-dimensional gravitational wave shown in Figure. 7.

Under the conditions of:

1. negligible viscous effect;

2. 2D planar wave only;

3. negligible gas density, or high density ratio between liquid and gas; and

4. linear small perturbation wave,

One can derive a dispersion relationship of [4]:

$$
\omega^{2}=g k+\frac{\sigma k^{3}}{\rho}
$$

where $\omega$ is the angular frequency, $\mathrm{g}$ is the gravitational acceleration, $\mathrm{k}$ is the wave number, $\sigma$ is the surface tension, and $\rho$ is the liquid density. With $\mathrm{k}=2 \pi / \lambda, \lambda$ as the wavelength, we can find the frequency as:

$$
f=\sqrt{\frac{g}{2 \pi \lambda}+\frac{2 \pi \sigma}{\rho \lambda^{3}}}
$$

It is seen that the wave frequency depends on surface tension, gravity acceleration, and wavelength. A critical wavelength can be found when the surface tension contribution is the same as that of gravity: 


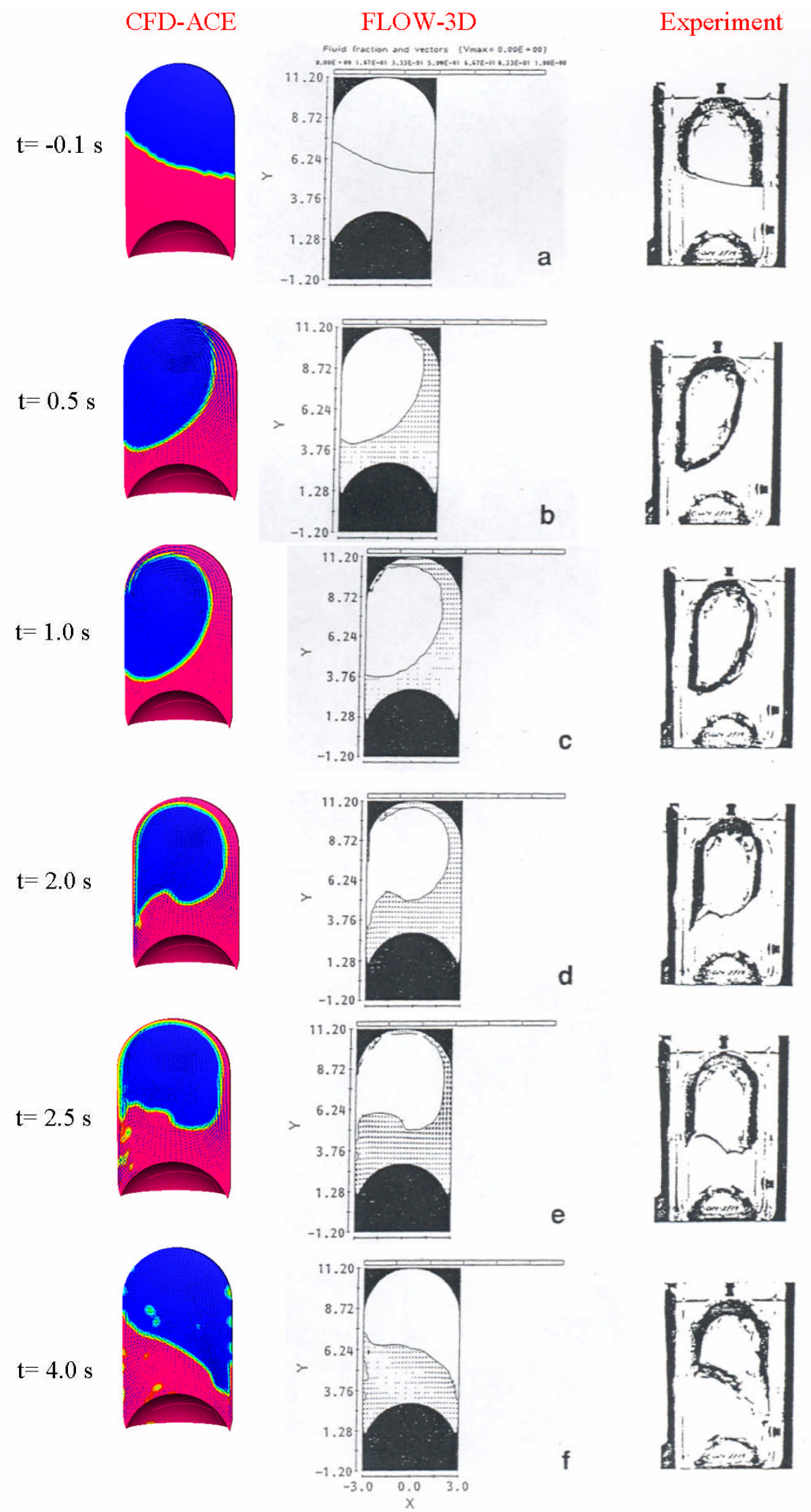

Figure 6. Comparison of Experimental and Computational Flow Field Results 


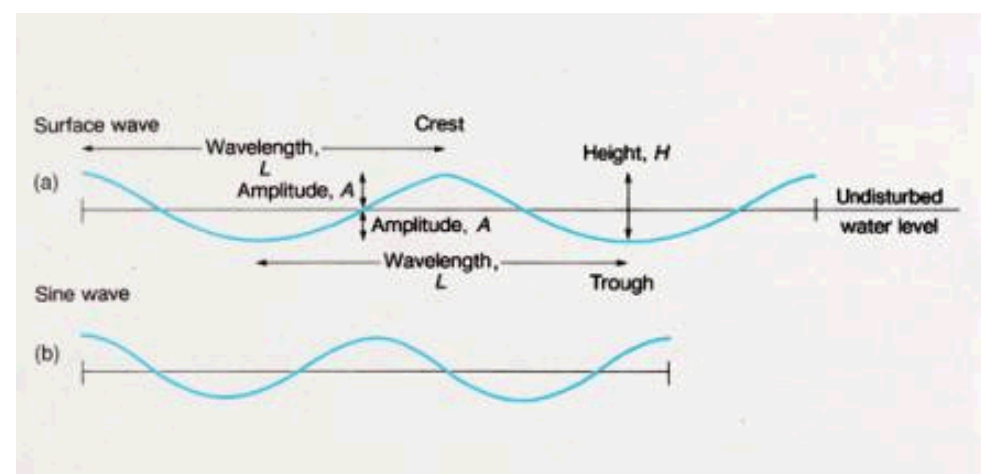

Figure 7. Surface Gravitational Wave on Liquid and Gas Interface

This gives that:

$$
\begin{gathered}
\frac{g}{2 \pi \lambda_{c}}=\frac{2 \pi \sigma}{\rho \lambda_{c}{ }^{3}} \\
\lambda_{c}=2 \pi \sqrt{\frac{\sigma}{\rho g}}
\end{gathered}
$$

On the ground with LH2 properties, one can find that $\lambda_{c}=0.01 \mathrm{~m}$

It indicates that surface tension is only important for very small ripples. For this reason, we can neglect the surface tension contribution. This leads to:

$$
f=\sqrt{\frac{g}{2 \pi \lambda}}
$$

To verify that a CFD simulation can accurately predict the natural frequency, a simplified model was made as shown in Figure 8.

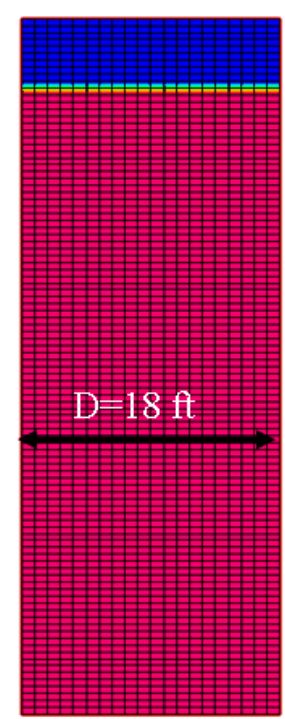

Simplified 2D Model (can be used to compare with theoretical solution)

\section{First Standing Mode} $\lambda=2 * \mathrm{D} \sim 36 \mathrm{ft}$

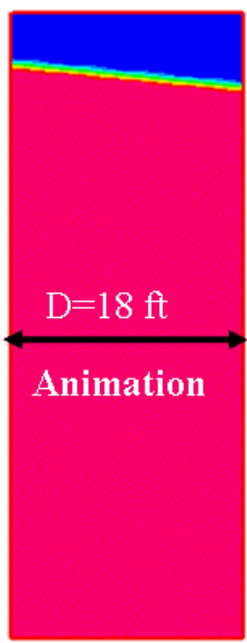

Theory: $f=0.3738$

Prediction: f_water $=0.3703$ f_LH $2=0.3623$
Second Standing Mode

$\lambda=\mathrm{D} \sim 18 \mathrm{ft}$

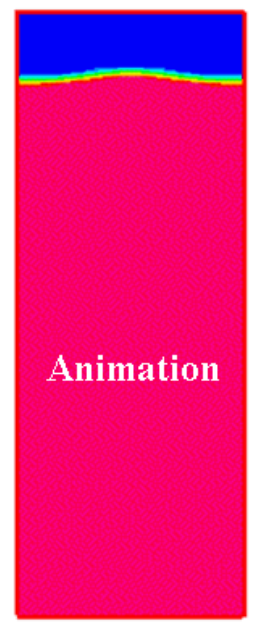

Theory: $f=0.5287$

Prediction: $f$ water $=0.5263$

f_LH $2=0.5$

Figure 8. Simplified two dimensional model to verify the theory

8

American Institute of Aeronautics and Astronautics 
First, the model is two dimensional planar, just as in the theoretical derivation. The simulation starts with an initial disturbed liquid surface profile either in the first mode or in the second mode (see Figure 8), and with the properties of water and air, due to their high density ratio. Once the waves settle down to their natural oscillation, the time required to complete a cycle is recorded, and the corresponding frequencies can be determined. The predicted first and second natural frequencies matched with the theory very well with errors less $1 \%$.

On the other hand, when using LH2 as liquid and gaseous hydrogen as gas, the natural frequency is slightly decreased in comparison to that of water and air as shown in Figure 8. This is very much expected, since the density ratio for LH2 is only about 10 , and the gas density contributes the wave motion in the form of added virtual mass. This will make a lower frequency: from $0.3703 \mathrm{~Hz}$ to $0.3623 \mathrm{~Hz}$ for the first mode, and from $0.5263 \mathrm{~Hz}$ to $0.5000 \mathrm{~Hz}$ for the second mode.

\section{Sloshing Frequency Validation Against Analytical Solutions of 3D Cylinder Tank}

Southwest Research Institute has been involved in "sloshing" research since before the start of the US space program. Dr. Norm Abamson, then the leader of the sloshing group has organized and published a monograph [5] which contained very comprehensive knowledge on both experimental and analytical results of sloshing and related subjects. The analytical solutions were derived for simple geometries such as rectangular boxes or the cylindrical tank with flat bottom face. These analytical solutions have been widely used to get mechanical parameters (mass, frequency, damping, mass center) for the preliminary design and for sloshing model input into space vehicles. In reality, the analytical results are applicable only to the simple geometries such as cylindrical and spherical tanks. CFD can be become a powerful tool in predicting these parameters for realistic propellant tanks after systematic validation. In this section, we will use the cylindrical tank to compare CFD predicted mechanical parameters against the analytical solutions.

\section{$\underline{\text { Simulation Model }}$}

The simulation model along with the computational grid is shown in Figure 9. Here a cylindrical tank with diameter $\mathrm{D}=3$ " and height of $\mathrm{H}=11$ " is modeled. The tank has no internal obstruction and all the walls are smooth. The top of the tank is open to ambient and is set up as fixed pressure boundary condition. All other boundaries are set as non-slip walls. There are a total of 80,000 cells, with 60 cells covering the whole tank diameter. This ensures that there will be at least 120 cells to resolve the first natural mode and 60 cells to resolve the second mode. Butterfly grid methodology is used to avoid the singularity at the tank center line, and to give better uniformity in the grid spacing. To determine the natural frequency of the tank slosh, an initial inclined surface is specified with a slope of $5.7 \mathrm{deg}$, as shown in Figure 9. A typical time dependent flow field and mass center coordinate of the liquid mass inside the tank, computed by integrating the Volume of the Fluid value in each cells, are shown in Figure 10.
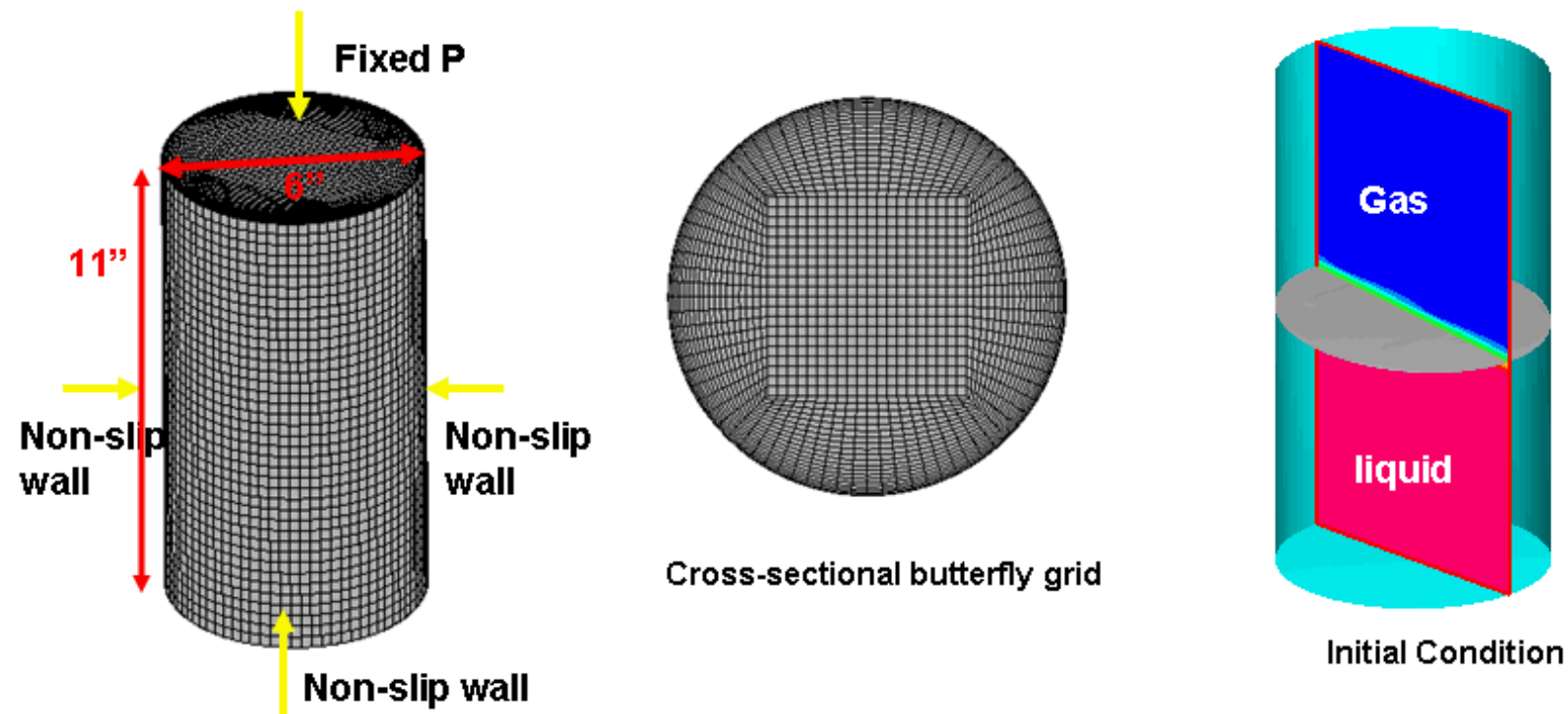

Figure 9. CFD model of a cylinder tank sloshing for comparison with analytical solutions. 

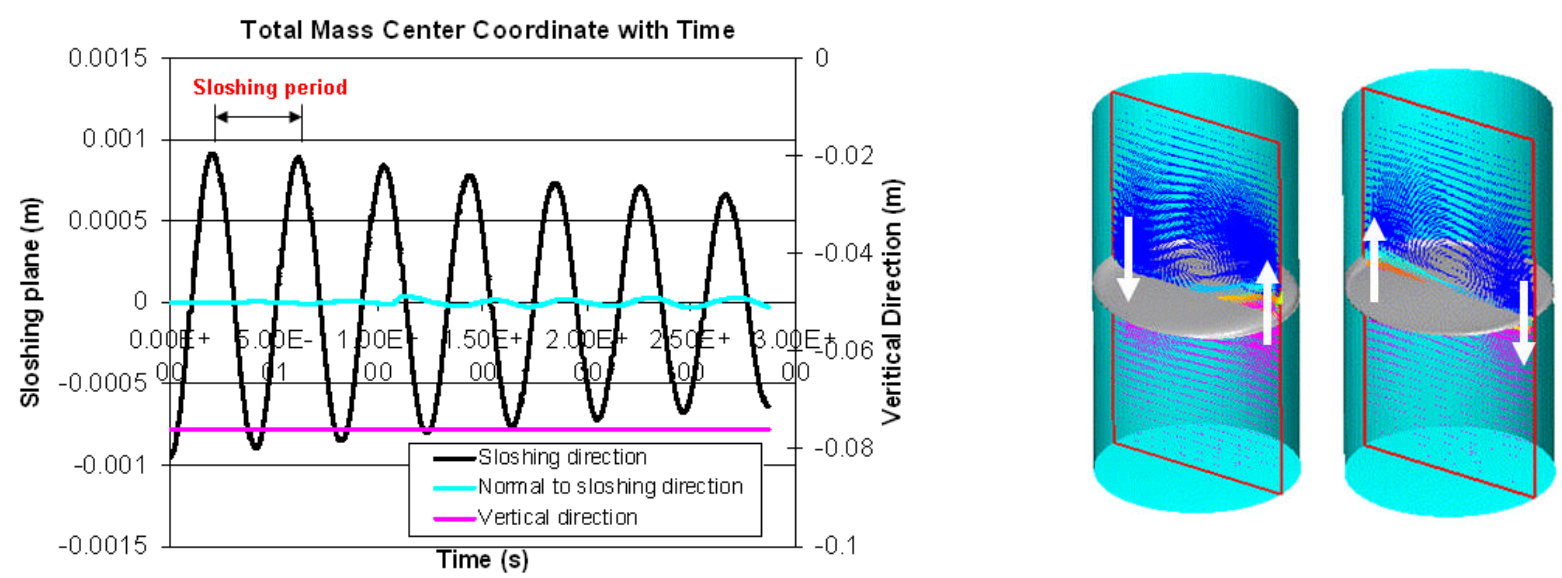

Figure 10. The center coordinates of the total liquid mass in the tank and flow field.

As seen from Figure 10, the vertical coordinate of the mass center is essentially constant, implying that the sloshing mass center does not move in the vertical direction, which is due to the symmetrical properties of surface wave. One may also notice almost zero value of the mass center in the direction normal to the sloshing plane. This indicates that sloshing is essentially two dimensional for this problem.

\section{Extraction of Natural Frequency}

From Figure 10 one can see that the sloshing has a rather constant period, which makes it possible to extract the natural frequency of the tank sloshing from the above data. Several simulations were made at different heights of the liquid level, and they are summarized in table 1. For the same configuration, the analytical solution of the natural frequency has been derived in [5], as

$$
f_{1}=\frac{1}{2 \pi} \sqrt{\frac{1.841 g}{R} \tanh \left(\frac{1.841 h}{R}\right)}
$$

Table 1. Sloshing Frequency Comparison for a Cylinder Tank in Water, $R=3$ "

\begin{tabular}{|c|c|c|c|c|}
\hline Liquid Level & $\mathrm{h} / \mathrm{R}=2.0$ & $\mathrm{~h} / \mathrm{R}=1.0$ & $\mathrm{~h} / \mathrm{R}=0.5$ & $\mathrm{~h} / \mathrm{R}=0.25$ \\
\hline Analytical & $2.4523 \mathrm{~Hz}$ & $2.3880 \mathrm{~Hz}$ & $2.0867 \mathrm{~Hz}$ & $1.6058 \mathrm{~Hz}$ \\
\hline Present CFD & $2.4539 \mathrm{~Hz}$ & $2.3753 \mathrm{~Hz}$ & $2.0920 \mathrm{~Hz}$ & $1.6155 \mathrm{~Hz}$ \\
\hline Error & $0.06 \%$ & $0.53 \%$ & $0.56 \%$ & $0.97 \%$ \\
\hline
\end{tabular}

The resulting frequencies at different liquid fill levels calculated from the above relationship are also listed in Table 1. It is seen that the present CFD predictions show excellent agreement with the analytical solution for all the liquid levels. All the discrepancies are less than $1 \%$. This builds the confidence of the CFD tool in predicting sloshing frequency.

\section{Sloshing Frequency Comparison with Experimental Data for Scaled Centaur Oxygen Tank}

An experimental investigation of liquid sloshing in a 1/3.75 scale-model Centaur stage liquid oxygen tank was conducted at NASA Lewis Research Center to determine (1) fundamental frequencies of oscillation, (2) the maximum horizontal slosh forces and damping ratios occurring at the fundamental frequencies, and (3) the slosh damping effectiveness of the proposed slosh baffle. Experimental data was obtained over a range of liquid depth ratios for the following tank configurations: (1) clean tank with no internal hardware, (2) unbaffled tank that included simulated internal flight hardware such as the trust distribution cylinder, and spring ring, and supporting structure in addition to the same internal hardware used in the unbaffled tank. The contained liquid was water in most tests. The results of this investigation were presented in terms of dimensionless parameters. 
The experimental test facility is shown in Figure 11, and the CFD simulation model is also shown in Figure 11. The three dimensional CFD model has 275,000 butterfly cells to remove the singularity near the tank center. The 1/3.75 scale model has a major diameter of axis of $32 \mathrm{inch}$, and minor diameter of $22.86 \mathrm{inch}$. A cylinder section with 0.88 inch height is located at the equator to simulate the cylindrical portion of the full scale tank.

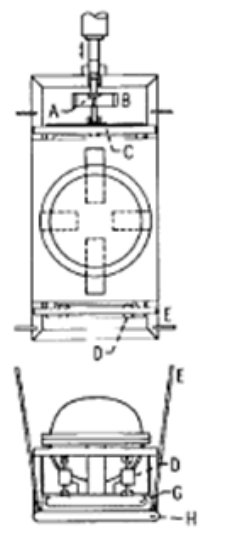

A. 5000-Pound prelaaded compression lasu cell . Prelosting tracket

C. 500-Pound tension-compression laud cell

b. 1000-Pound tension-compression laad cell

E. Support members from overneas crossbeams to frame

. 32 -Inch-diameter spheroidal tank

G. Test bes

t. Hydraulic cylinder

i. Stationary support

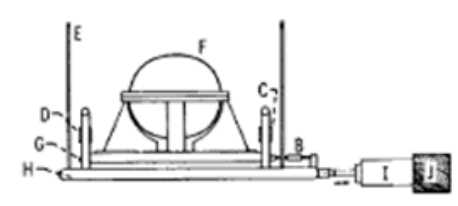

(a) Schenatic view.

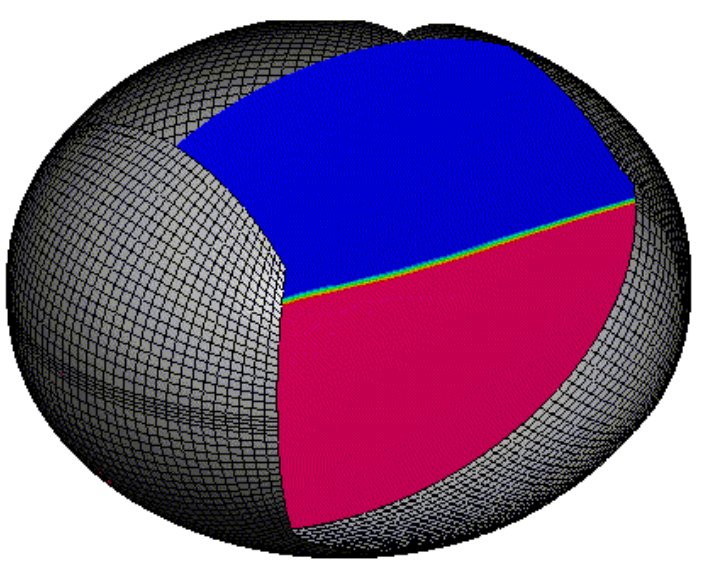

Test Facility

CFD Simulation Model

Figure 11. Experimental test facility and CFD simulation model for the Centaur liquid oxygen tank.

Natural Frequency Comparison for Smooth Tank

During the test, the tank was forced to oscillate sinusoidally at a preselected excitation frequency and amplitude. At each liquid depth ratio investigated, the excitation frequency chosen was equal to the fundamental frequency of the oscillation of the contained liquid to provide the maximum slosh forces at a given excitation amplitude. When the first mode waveform has built up to its maximum height on the tank wall, the oscillatory motion of the tank was quick-stooped, and the residual horizontal slosh forces were recorded on an oscillograph trace. The fundamental frequencies of the liquid oscillation were determined by using the first several slosh force peaks occurring immediately after the quick stop, and was put into the form of the fundamental frequency parameter:

$$
\eta=\omega \sqrt{\frac{r}{g}}
$$

where $\mathrm{r}$ is the radius of liquid surface of partially filled oblate spheroidal tank, and $\mathrm{g}$ is the gravitational acceleration $\left(9.8 \mathrm{~m} / \mathrm{s}^{2}\right)$.

Both the experimentally determined frequency parameter and those from CFD modeling are given in Figure 12. As one can see, excellent agreements were found between the present CFD prediction and the experimental measurement. 


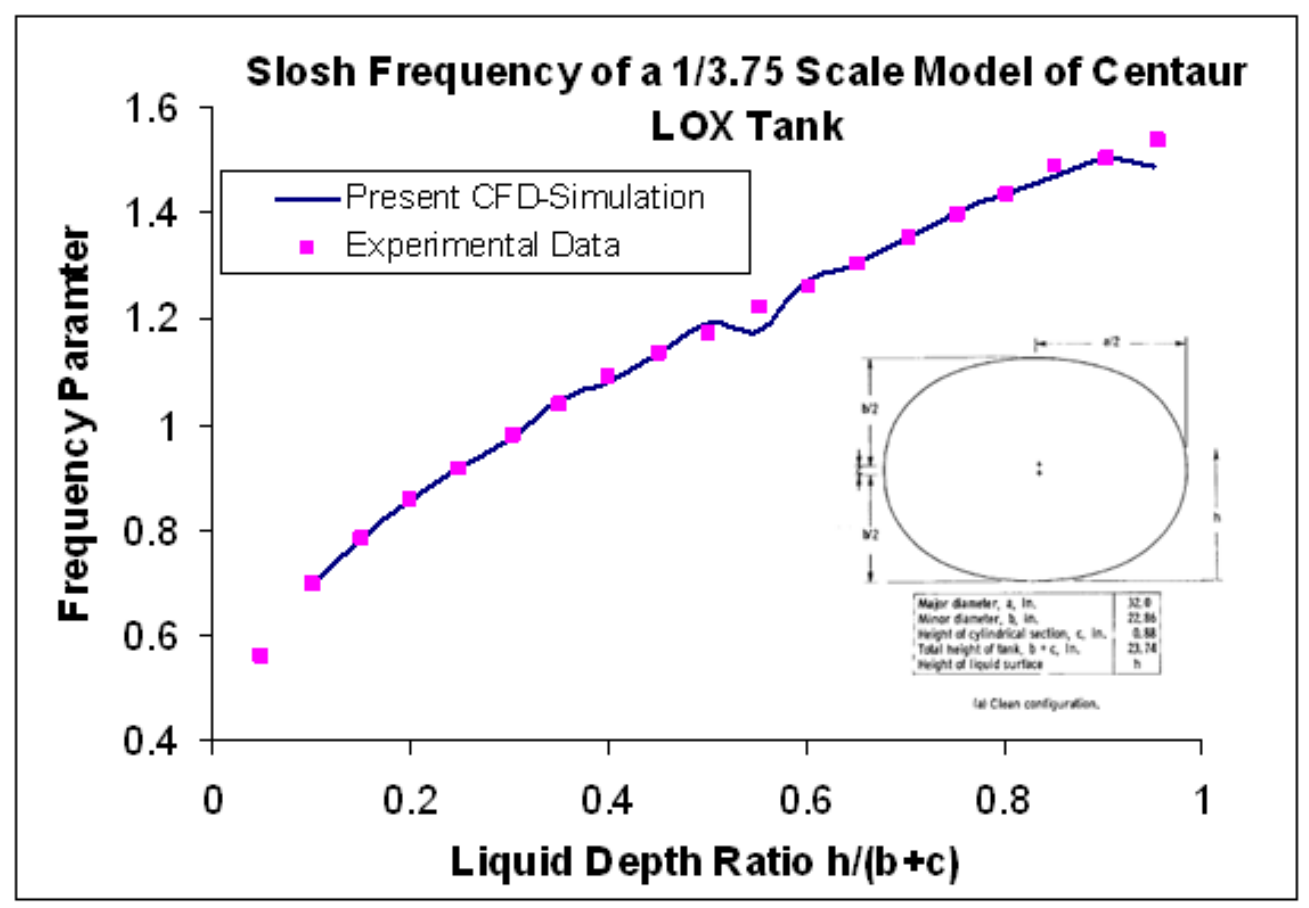

Figure 12. Comparison of CFD extracted frequency vs. experiments for 1/3.75 scale model of Centaur LOX Tank.

\section{E. Sloshing Frequency Comparison with Experimental Data for Scaled Centaur Hydrogen Tank}

Centaur hydrogen tank is different from the oxygen tank in that it has a cylindrical top and an oblate spheroidal common bulkhead with the oxygen tank. The geometry of the tank along with the test facility is shown in Figure 13 . Sumner et al [7] measured the fundamental frequencies of the liquid oscillations, sloshing force and damping ratio for both the unbaffled and the baffled tank configurations for a range of liquid levels above the top of the concave oblate spheroidal tank bottom. This configuration is unique in that no analytical solution can be derived for the exact same geometry. Tests had been done for the case with a baffle located at liquid depth ratio of 0.59 , and this is a good validation case for CFD model in capturing baffle impact.
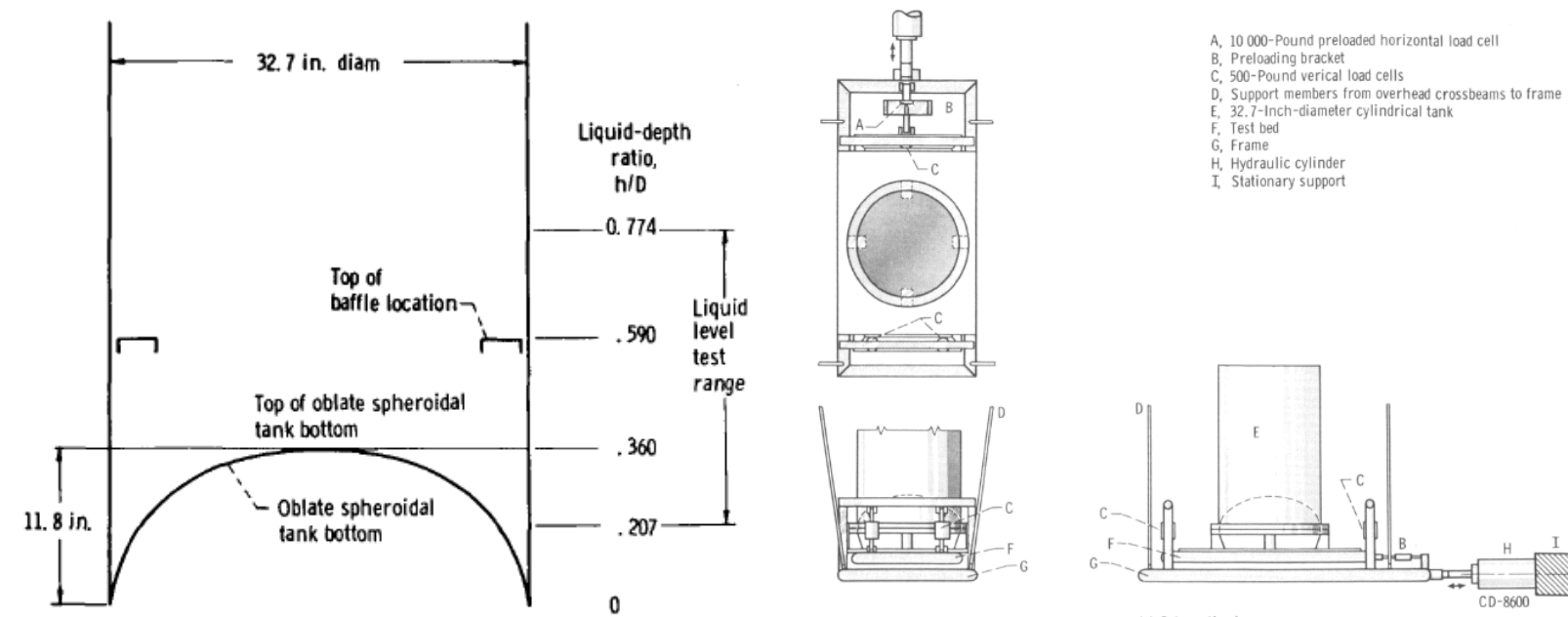

Figure 13. Schematic of scaled-model Centaur liquid-hydrogen tank and test facility

The pictorial view of the hydrogen tank and its model using CFD tool are shown in Figure 14. A baffle of 12

American Institute of Aeronautics and Astronautics 
2.19" wide is located at liquid depth ratio of 0.59. The tank is discretized with a total of 410,000 cells.

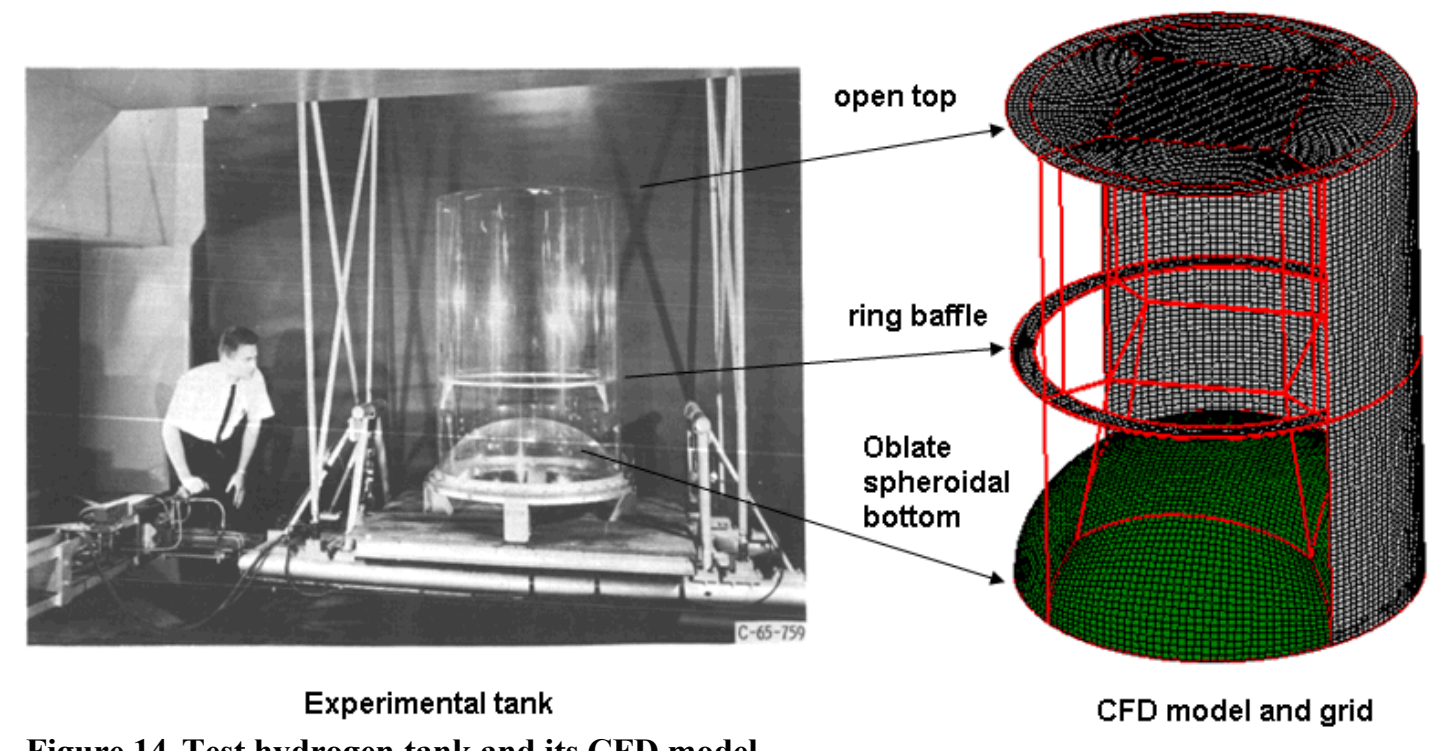

Figure 14. Test hydrogen tank and its CFD model

Natural Frequency for Smooth Tank

The test procedure to obtain the fundamental frequency of slosh was similar to that for the oxygen tank. First, the tank was oscillated sinusoidally at a preselected excitation frequency (from $0.3 \mathrm{~Hz}$ to $4.8 \mathrm{~Hz}$ ) and amplitude. At each liquid depth ratio (see Figure 14), the excitation frequency chosen was equal to the actual fundamental frequency of the slosh to provide the maximum slosh force. When the waveform had built to its maximum height on the tank wall, the oscillation of the tank was quick-stopped, and the residual horizontal slosh forces were recorded on the oscillograph trace. The first natural frequency of the liquid oscillation was then determined by using the first several slosh-force peaks occurring immediately after the quick stop. The test data, CFD extracted frequency, and the analytical solution based on a straight cylinder are shown in Figure 15. The analytical solution based on the straight cylinder solution over-predicts the frequency parameter for the liquid depth ratio from 0.45 to 0.8 . When the liquid depth ratio is above 0.8 , the curved bottom effect can be neglected and a straight cylinder solution can be applied. On the other hand, the present CFD simulation can model different geometrical configurations of the tank, and shows very favorable comparison with the test data as seen from Figure 15. This validation case illustrates the capability of CFD in extracting mechanical parameters for complicated tank geometries when there is no analytical solution available.

\section{Natural Frequency for Hydrogen Tank with Baffle}

Next we consider the case when there is a baffle inside the tank. The baffle has a width of 2.9 " and is located at liquid depth ratio of 0.59. Figure 16 illustrates the sloshing interface dynamics during CFD simulations at several liquid depth ratios. The comparison of natural frequencies from the present CFD and test data is given in Figure 17. The experimental test showed that the presence of the baffle sharply increased the natural frequency parameter when the liquid-depth ratio corresponded to the baffle location, and the baffle reduced the frequency parameter when the liquid level was slightly above the baffle location. This increase in frequency can be understood as the reduction of cross-sectional radius of the tank due to the existence of the baffle when the liquid level is at the baffle location. In general CFD simulation shows fair agreement with the test, including the sudden rise and fall observed in the test near the baffle location. Even though the predicted amplitude of rise and fall is not as dramatic as the test data, the fundamental physics of the baffle impact on the natural frequency is well captured in the present modeling. Systematic study is under way to investigate the discrepancy. 


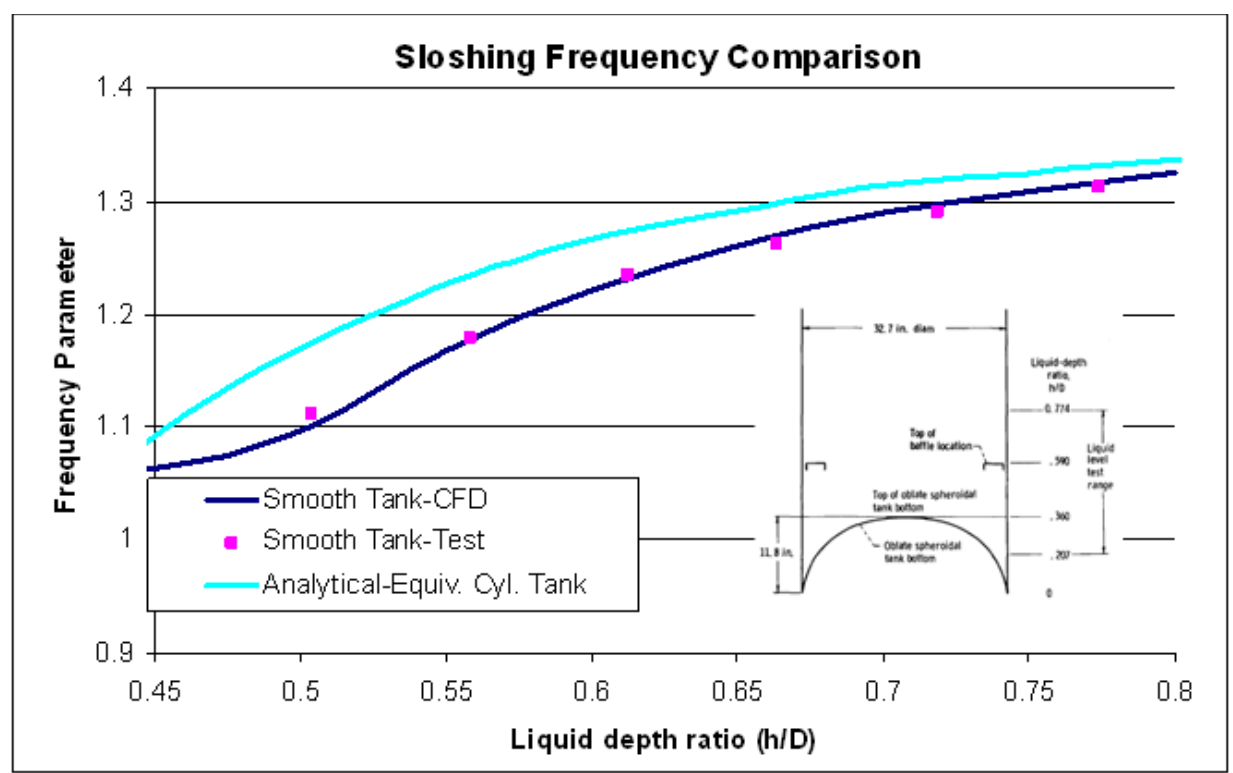

Figure 15. Comparison of sloshing natural frequencies for the scale-model of Centaur hydrogen tank

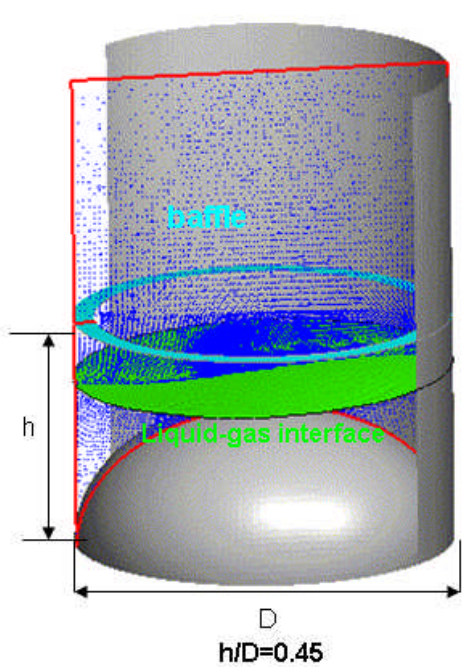

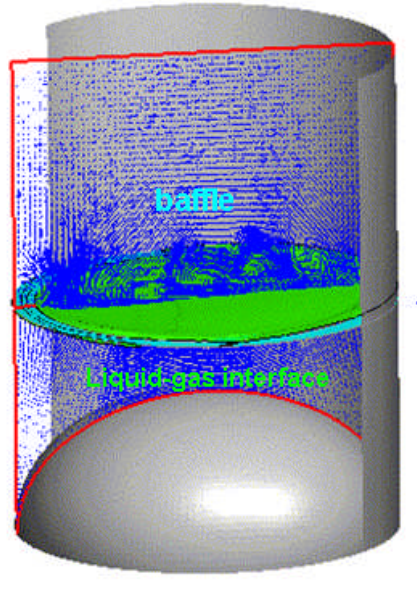

$h / D=0.60$

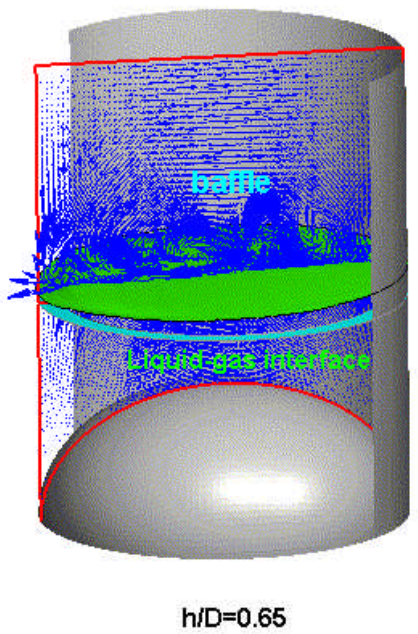

Figure 16. CFD free interface dynamics at different liquid depth ratios inside scale model of Centaur hydrogen tank. 


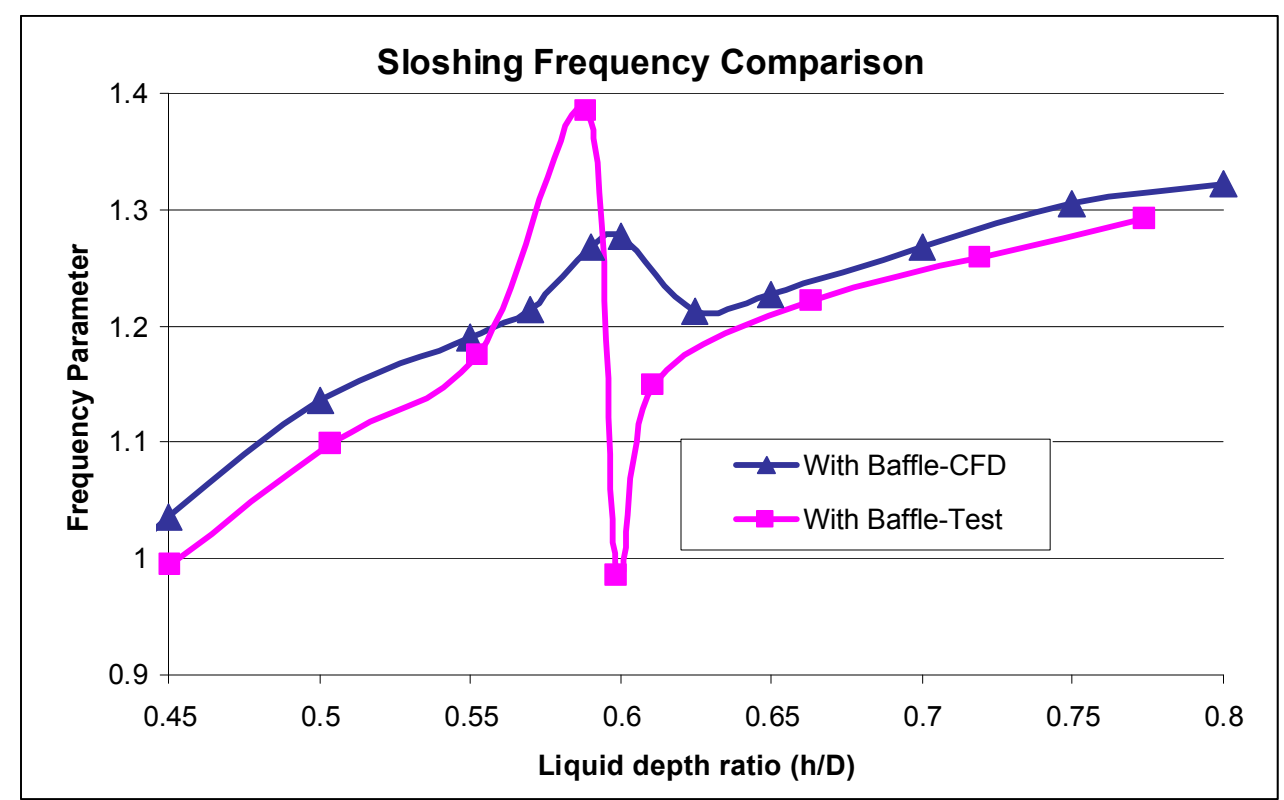

Figure 17. Comparison of sloshing natural frequencies for the scale-model of Centaur hydrogen tank with a baffle

\section{F. Sloshing Mass Extraction Validation}

The above sections describe the effort in extracting fundamental frequency of the slosh from a CFD simulation. All results show good agreement in comparison with experimental data. For the mechanical model, one also needs the slosh mass and its center. In the experiment of reference [6], the tank was forced to oscillate at different amplitude to derive the wave amplitude of the liquid surface as high as possible. Once the wave height had reached a steady-state value, the oscillatory motion of the tank was quick-stopped, and the horizontal slosh force was determined from the first force peak occurring immediately thereafter to calculate the slosh mass. The similar procedure that can be used in CFD modeling to determine the slosh mass is by forcing the tank to a periodic motion with amplitude of body acceleration $\alpha \mathrm{g}$ :

$$
m_{s} \frac{d^{2} x_{s}}{d t^{2}}+k_{s} x_{s}=-m_{s} \alpha g \sin \left(2 \pi \omega_{f} t\right)
$$

where $\omega_{\mathrm{f}}$ is the forcing frequency. Under the steady state condition, the slosh mass will oscillate at a periodic state with a frequency the same as the forcing frequency $\omega_{\mathrm{f}}$. Let:

$$
x_{s}=x_{s o} \sin \left(2 \pi \omega_{f} t\right)
$$

Noting that:

$$
x_{t}=\frac{m_{s}}{m_{t}} x_{s} \text { and } \omega_{n}{ }^{2}=\sqrt{\frac{k_{s}}{m_{s}}}
$$

we can find that: 


$$
x_{s o}=\frac{\alpha g}{\left(\omega_{n}{ }^{2}-\omega_{f}{ }^{2}\right)}
$$

Using equation (18), we can find that:

$$
\frac{m_{s}}{m_{t}}=\frac{x_{t o}}{\alpha g}\left(\omega_{n}{ }^{2}-\omega_{f}{ }^{2}\right)
$$

Here, $\mathrm{x}_{\mathrm{to}}$ is the maximum mass center displacement during the cycle. Using this method, there is no need to compute the force acting on the tank walls. As shown in Figure 18, first a sinusoidal body force is applied to the cylinder tank of Figure 9. The liquid fill level is at $\mathrm{h} / \mathrm{R}=2.0$, and the natural frequency predicted from the above simulation and analytical solution is $2.45 \mathrm{~Hz}$. The forcing frequency is set at $1 \mathrm{~Hz}$, and the total mass center history in Figure 18 shows an initial transient and then a periodic motion matching with a forcing frequency. The maximum value of the mass center displacement is the value used for equation (20).

Figure 18. Procedure to extract slosh mass and the sample mass center history

Validation of Slosh Mass against Analytical Solution

For the cylinder tank, the analytical solution of the sloshing mass to total tank mass ratio has been derived in [5], as:

$$
\frac{m_{s}}{m_{t}}=\frac{0.4547 R}{h} \tanh \frac{1.84 h}{R}
$$

Table 2 shows the comparison of CFD prediction and analytical solution on the slosh mass ratio. Again, one can clearly see the excellent agreement with error less than $0.6 \%$. 
Table 2. Sloshing Mass Ratio (m1/m) Comparison for a Cylinder Tank in Water, $R=3$ ”

\begin{tabular}{|c|c|c|c|c|}
\hline Liquid Level & $\mathrm{h} / \mathrm{R}=2.0$ & $\mathrm{~h} / \mathrm{R}=1.0$ & $\mathrm{~h} / \mathrm{R}=0.5$ & $\mathrm{~h} / \mathrm{R}=0.25$ \\
\hline Analytical & 0.22735 & 0.43236 & 0.66028 & 0.78252 \\
\hline Present CFD & 0.22858 & 0.43380 & 0.66402 & 0.77950 \\
\hline Error & $0.54 \%$ & $0.33 \%$ & $0.56 \%$ & $0.39 \%$ \\
\hline
\end{tabular}

\section{Conclusion}

The present study represented a continuing effort in validating CFD technology in modeling space vehicle sloshing dynamics $[8,9]$. This effort has clearly illustrated the soundness of the CFD approach in modeling the detailed fluid dynamics of tank sloshing and the excellent accuracy in extracting mechanical properties for different tank configurations and at different fill levels. The present study shows that CFD technology is especially applicable to the future design of propellant tanks, as there is no previous experimental data available for the same size and configuration. From the geometrical point of view, the analytical solution is only available for the straight cylinder. It is good fit for the LH2 tank when the liquid level is high. CFD modeling has no limitation, and can take the real geometry into account. The analytical solutions are generally obtained for the linear analysis, where the amplitude of the slosh wave is small. CFD solution can go beyond the linear stability regime, and predict what will happen after the surface breakup. CFD simulation has also no limitation on the gravitational acceleration, and can be applied to the realistic flight modeling.

Extracting the realistic damping ratio is a much challenge task as the fluid damping depends greatly on the grid resolution, numerical scheme and the ability in identifying the boundary thickness $a$ prior. This is especially true when there exists a baffle. The results of the validation study will be reported in the future.

\section{References}

${ }^{1}$ H. Q. Yang, and A. J. Przekwas, "Computational Modeling of Microfluid Devices with Free Surface Liquid Handling", Technical Proceeding of the 1998 International Conference on Modeling and Simulation of Microsystems, MSM 98, pp. 498-505, 1998.

${ }^{2}$ L. E. Toole and L. J. Hastings, "An Experimental Study of the Behavior of a Sloshing Liquid Subjected to a Sudden Reduction in Acceleration", NASA TM X-53755, 1968.

${ }^{3}$ M. F. Fisher, G. R. Schmidt and J. J. Martin, "Analysis of Cryogenic Propellant Behaviour in microgravity and low thrust environments", paper presented at the 1991 space Cryogenics Workshop, Jun 2, 18-20, 1991, Cleveland, $\mathrm{OH}$, USA.

${ }^{4}$ V. G. Levich, Physicochemical Hydrodynamics, Prentice-Hall, New Jersey, Chapter \# XI, 1962.

${ }^{5}$ H. N. Abramson, "The Dynamic Behavior of Liquids in Moving Containers", NASA SP-106, 1967.

${ }^{6}$ I. E. Sumner, A. J. Stofan and D. J. Shramo, "Experimental Sloshing Characteristics and a Mechanical Analogy of Liquid Sloshing in a Scale-Model Centaur Liquid-Oxygen Tank", NASA TM-X-999, August, 1964.

${ }^{7}$ I. E. Sumner, R. F. Lacovic, and A. J. Stofan, "Experimental Investigation Of Liquid Sloshing In A Scale-Model Centaur Liquid-Hydrogen Tank", NASA TM-X-1313, November, 1966

${ }^{8}$ H. Q. Yang, J. M. Rojahn, J. W. Peugeot, D. J. Dorney. "CFD Analysis of Propellant Tank sloshing under Vertical Oscillatory Thrust and Horizontal Oscillatory Side Load", JANNAF 2008, Paper LPS-06, Feed System Design and Analysis, Orlando, November 10-13, 2008.

${ }_{9}$ H. Q. Yang and J. W. Peugeot, "Characteristics of Surface Waves Under Thrust Oscillation”, JANNAF 2010, Paper LSP-1154, Feed System Design and Analysis, Colorado Spring, CO, May 3-7, 2010. 